OPEN ACCESS

Edited by:

Xu-jie Zhou,

Peking University First Hospital, China

Reviewed by:

Gleb Radchenko,

South Ural State University, Russia

Koen R. C. Bruynsee/s,

Delft University of Technology,

Netherlands

${ }^{*}$ Correspondence:

Tjalf Ziemssen

tjalf.ziemssen@uniklinikum-dresden.de

Specialty section: This article was submitted to Autoimmune and

Autoinflammatory Disorders, a section of the journal

Frontiers in Immunology

Received: 19 February 2021

Accepted: 16 April 2021

Published: 03 May 2021

Citation:

Voigt I, Inojosa $\mathrm{H}$

Dillenseger A, Haase R, Akgün K and Ziemssen T (2021) Digital

Twins for Multiple Sclerosis.

Front. Immunol. 12:669811. doi: 10.3389/fimmu.2021.669811

\section{Digital Twins for Multiple Sclerosis}

\author{
Isabel Voigt, Hernan Inojosa, Anja Dillenseger, Rocco Haase, Katja Akgün \\ and Tjalf Ziemssen * \\ Center of Clinical Neuroscience, Department of Neurology, University Hospital Carl Gustav Carus, Technical University of \\ Dresden, Dresden, Germany
}

An individualized innovative disease management is of great importance for people with multiple sclerosis (pwMS) to cope with the complexity of this chronic, multidimensional disease. However, an individual state of the art strategy, with precise adjustment to the patient's characteristics, is still far from being part of the everyday care of pwMS. The development of digital twins could decisively advance the necessary implementation of an individualized innovative management of MS. Through artificial intelligence-based analysis of several disease parameters - including clinical and para-clinical outcomes, multi-omics, biomarkers, patient-related data, information about the patient's life circumstances and plans, and medical procedures - a digital twin paired to the patient's characteristic can be created, enabling healthcare professionals to handle large amounts of patient data. This can contribute to a more personalized and effective care by integrating data from multiple sources in a standardized manner, implementing individualized clinical pathways, supporting physician-patient communication and facilitating a shared decision-making. With a clear display of pre-analyzed patient data on a dashboard, patient participation and individualized clinical decisions as well as the prediction of disease progression and treatment simulation could become possible. In this review, we focus on the advantages, challenges and practical aspects of digital twins in the management of MS. We discuss the use of digital twins for MS as a revolutionary tool to improve diagnosis, monitoring and therapy refining patients' well-being, saving economic costs, and enabling prevention of disease progression. Digital twins will help make precision medicine and patient-centered care a reality in everyday life.

Keywords: multiple sclerosis, precision medicine, personalized medicine, digital twin, decision analysis, medical care

\section{INTRODUCTION}

The technology of digital twins (DTs) is a promising concept that has become the focus of interest in industry and, in recent years, in healthcare sector as well. DTs are a revolutionary tool in phenotyping patients, where analysis of large amounts of data (big data) through new technologies like artificial intelligence (AI) enables visualization of a virtual copy (twin) of the patient at different stages of the disease and supports further therapeutic decisions. However, the use of DTs in medical care and especially in the management of patients is still in its infancy. DTs have enormous potential, especially when it comes to precision medicine: they can be used to simulate individual therapies in advance and visualize potential therapy results and disease progression. The 
concept of DTs seems to be particularly suitable for the treatment of multiple sclerosis (MS), because this chronic autoimmune "disease of a thousand faces" is characterized by heterogeneous course, complexity and multidimensionality, an increasing number of treatment options and a resulting wealth of data. DTs can significantly improve precision medicine for people with MS (pwMS) by enabling healthcare professionals (HCPs) to handle big data and provide more personalized and effective care. In this paper, we focus on our vision of how to design a DT for the management of MS. The advantages of DTs for the personalized treatment of individual pwMS are highlighted without ignoring the challenges on its development. With our review, we want to answer the question whether "Digital Twins for Multiple Sclerosis" (DTMS) may serve as a game changer in the management of MS.

\section{MULTIPLE SCLEROSIS REQUIRES PRECISION MEDICINE}

\section{Multiple Sclerosis as a Chronic Multidimensional Disease}

MS is a chronic autoimmune, degenerative and lifelong disease of the central nervous system (CNS) and the most common cause of neurological disability in young adults. At a pathological level, the infiltration of immune cells into the CNS manifests as localized demyelinating lesions in the white and gray matters of the brain and spinal cord, observed in pathological specimens as well as in magnetic resonance imaging (MRI) sequences (1). In addition, the disease leads to a progressive destruction of myelin layers (demyelination) and progressive axonal injury, loss and neurodegeneration, impairing the function of the CNS in several ways $(2,3)$.

MS has different clinical disease courses that have been classically described. Around $85-90 \%$ of the patients are diagnosed with a relapsing remitting form of the disease (RRMS) at the beginning $(4,5)$. These patients are affected by attacks of unpredictable clinical relapses caused by inflammatory demyelinating lesions in the CNS, resulting in a complete or partial recovery of the neurological symptoms. After several years, the majority of these patients if untreated will develop secondary progressive MS (SPMS), where the neurological function decreases over time independent of relapse activity $(6,7)$. About $10-15 \%$ of the patients do not have relapses during the course of the disease. In these patients, the disease already begins with a gradual increase in neurological symptoms. This is called primary progressive progression (PPMS). Often a spastic gait disorder develops over the years, more rarely a progressive cerebellar syndrome (8). Beyond this raw classification of disease courses, each MS patient presents with a very individual course of his MS.

Longitudinal course. As described, MS is characterized by a chronic and/or episodic course. PwMS require long-term phenotyping, monitoring and most often treatment with disease-modifying therapies (DMTs) (9). In the early stages of MS, the damage occurring in the brain can still be compensated by the so-called neurological reserve. This compensatory mechanism explains why, on the one hand, early-stage MS is often not diagnosed promptly and, on the other hand, is often not taken seriously enough, especially with regard to negative long-term consequences (10-12). As the disease progresses, the neurological reserve decreases, especially if MS activity is not adequately treated (12). Since therapy started early in the course of the disease has an inhibitory effect on the progression of MS, it should be diagnosed and treated without any delays $(13,14)$.

Heterogeneous course and different dimensions. MS is popularly known as the "disease of a thousand faces" because MS lesions and other abnormalities can occur in the whole CNS usually leading to a variety of neurological deficits including fatigue, visual and bladder problems, pain, spasticity, reduced mobility and sexuality as well as psychological conditions such as depression (15-17). Due to this heterogeneity and the intraindividual unpredictable and inter-individually quite variable course, the diagnosis, phenotyping and monitoring of MS is very challenging $(18,19)$. The multidimensional disease characteristics of each patient should be made quantifiable to allow phenotyping of the individual disease characteristics and long-term monitoring of these parameters (20). This leads to a large amount of multidimensional data.

Multidimensional data. When quantifying MS, it is necessary to distinguish between different dimensions and perspectives. Starting from neurological-clinical parameters, they range from quantitative assessment of individual neurological functional systems (e.g. cognition, gait analysis), through imaging (MRI, ocular coherence tomography (OCT)), electrophysiological methods and the inclusion of patientreported outcomes (PRO), up to new molecular and digital biomarkers (20). This data can be obtained in the setting of clinical trials or in real world practice, which represents also differences in its collection, volume, veracity and availability. To do justice to the complexity of MS, these parameters must be integrated into detailed individual patient charts as well as into large databases in order to be able to analyze them meaningfully.

Increasing number of potential therapeutic interventions. The number of treatment options that intervene in the immune system on different levels can modify disease is increasing (19, 21-25). This growing availability of DMTs is broadening the treatment options towards a more individualized therapy (24). Different mechanisms of action and intervention strategies are linked to individual treatments (26-29). On treatment, the monitoring of MS disease activity is key to achieve optimal outcomes in order to initiate a therapy change or escalation in time in case of an insufficient response $(10,30)$.

Therefore, the chronic, heterogenic and multifocal "disease of a thousand faces" requires a complex, ubiquitous and differentiated as well as adaptive diagnosis, monitoring and treatment strategy. This strategy should be personalized and tailored to the individual needs and disease course of the patient and be continuously adjusted (25).

\section{Precision Medicine for People With Multiple Sclerosis}

An emerging approach towards personalized treatment is precision medicine, or, as an older term, personalized medicine 
(31-35), that takes into account individual variability in genes, environment, and lifestyle for each person (32, 36-42). Precision medicine covers diagnosis, treatment and management to achieve better patient outcomes (43). Through precision medicine, it is possible to break down the complexity of the disease. The patterns and inter-individual variability can be better understood. Thereby, precision medicine presents a framework for developing targeted treatment for individual patients by combining the demographic and clinical information, biomarkers and medical imaging data (44-47). Existing developments in precision medicine (44, 48-50) demonstrate that complex health-related big data of high quality are necessary, including lifestyle, nutrition, genetics, and environmental factors besides clinical, para-clinical, imaging and immunological or neurobiological parameters, which have to be analyzed and integrated in diagnosis, treatment and monitoring processes. To obtain big data and capture the bigger picture of a given individual on the way to precision medicine, Fagherazzi et al. recommend the method of "deep digital phenotyping", which is a combination of deep phenotyping by collecting biomedical data in the real world and digital phenotyping by collecting digital biomarkers $(42,44,51-53)$.

In the patient's perspective, a more transparent disease understanding can enable the patient to take a more active role in decision-making, following the concept of patient empowerment (54). Better understanding and involvement of patients in therapeutic decision making leads to better treatment adherence, which is associated with higher efficacy and lower healthcare costs (55). Ultimately, all patients would have the opportunity to query their own data interpreted in the context of the world's largest reference cohort and the latest data on available therapeutic options (56).

In relation to $\mathrm{MS}$, deciding which therapy to use in a particular patient requires careful analysis of the patient's disease course for high-risk factors for early progression, consideration of the efficacy and safety profile for a potential therapy, and a patient's lifestyle and expectations (57). This is the only way to improve the precision of management for each patient, to improve prognosis and to establish an evidence-based framework for predicting response to treatment and personalized monitoring of patients. Precision medicine for pwMS involves the classification of disease subtypes based on underlying biology, not just clinical phenotype, and the development of predictive models that incorporate the integration of clinical, biological and molecular as well as current and emerging imaging markers with an understanding of the impact of the disease on the lives of individual patients (58-63). A complex data set could be the base of the DTMS as part of a digital data cloud that tries to simulate the same or very similar characteristics in terms of health status, risk factors and disease development as the real-world MS patient $(43,45)$.

\section{WHAT ARE DIGITAL TWINS?}

\section{Origin and Concept Of Digital Twins}

The concept of a "twin strategy" was generated from NASA's Apollo program, which build two real identical space vehicles.
One was launched onto the air space, the other stayed on Earth to mirror the conditions of the launched one (64). The first mention of the term "digital twin" can be traced back to the year 2003 when Grieves mentioned it in the context of manufacturing (6466). Initially, the space industry was primarily concerned with the topic of DT. In 2012, the NASA and the U.S. Air Force jointly published a paper about the DT, which stated the DT was the key technology for future vehicles. After that, the number of research studies on DT in aerospace has increased and the DT was introduced into more fields such as automotive, oil and gas as well as health care and medicine. Examples are online operation monitoring of process plants, traffic and logistics management, dynamic data assimilation enabled weather forecasting, real-time monitoring systems to detect leakages in oil and water pipelines, and remote control and maintenance of satellites or spacestations. For instance, Singapore is developing a digital copy of the entire city to monitor and improve utilities (67). In recent years, the DT has been described more and more as a promising technology and it is expected that DTs will develop very strongly in the coming years and will bring a revolution in several industry sectors with the desire for online monitoring, increasing flexibility and personalized services (64). The availability of cheap sensors and communication technologies and the phenomenal success of technologies such as machine learning (ML) and AI, new developments in computer hardware as well as cloud and edge computing will rapidly drive the development of the DT (66).

Grieves (65) originally defined the DT in three dimensions: a physical entity, a digital counterpart and a connection that ties the two parts together. In most definitions, the DT is considered as a virtual representation that interacts with the physical object throughout its lifecycle and provides intelligence for evaluation, optimization, prediction, etc. (68-72). For instance, in the industrial sector the DT is used as an in silico presentation of technical applications in order to optimize them through computer simulations $(67,73,74)$. As these definitions focus on three dimensions (physical, virtual, connection of them), Tao et al. added the two further dimensions data and services. The newly proposed definition can fuse data from both the physical and virtual aspects using DT data for more comprehensive and accurate information capture (64). Kritzinger et al. divide DT into three subcategories, depending on the level of data integration (75). Rasheed et al. present an example of a stateof-the-art DT of an offshore oil platform. The DT is continuously updated with sensor data almost in real time. The sensor data can be supplemented with synthetic data from simulators that provide physical realism at high spatio-temporal resolution. The DT not only provides real-time information for more informed decision-making, but can also make predictions about how the plant will develop or behave in the future. In an ideal environment, a DT is indistinguishable from a physical object in both appearance and behavior, with the added benefit of being able to make predictions about the future. In fact, the DT also offers the possibility for people to physically interact with the object using an avatar (66).

Overall, it must be noted that the topic of DTs is of such variety and complicated that it is almost impossible to cover all 
aspects as it has been covered by several reviews (66, 76-88). Up to now, there are currently no common methods, standards or norms for the development of DTs. In order to exploit the potential of DTs, there are still many challenges to be taken $(66,67)$.

\section{Digital Twins in Health Care}

Focusing on the possibilities of DTs, medicine and healthcare are the areas that are likely to benefit most from the concept of DTs (66). There are several reasons for this. First, the number of intelligent portable devices and the organized storage of big data of individuals and cohorts is increasing. Second, human and thus medical thinking will eventually reach the natural limits of speed, complexity and performance. For HCPs, the massive and constant increase of knowledge in healthcare (e.g., differentiated diagnostics, more personalized therapies, interaction risks, active ingredients) is almost impossible to cope within daily work. HCPs are limited by everyday circumstances such as tiredness, time pressure and emotions. Especially in hospitals HCPs are under cost and time pressure and cannot always make decisions based solely on medical factors. And third, there is an increasing need for personalized and targeted treatment. As a result, various tools that enable precision medicine and simulation of therapies as well as prognosis of disease progression will inevitably find their way into the everyday life of HCPs, as is the case for already established different (clinical) decision support systems (CDSS) (89). The integration of technology and medicine is thus the main driver for intelligent and networked health. In this context, the statistical modeling of big data poses a particular challenge. Classical methods that examine associations between individual variables and a diagnosis or a course of disease reach their limits with the large number of statistical tests required and are also unable to uncover complex interactions between several variables and modalities in real time. Statistical significance, until now the primary measure of group-based, mechanistic research, also loses significance when, due to large samples, even the smallest effects exceed the significance threshold and thus the connection between significance and (clinical) relevance fades. ML is the key to creating direct clinical benefit. ML involves algorithms that can learn to solve a specific task autonomously based on data. These algorithms do not need to be explicitly programmed and can thus generate novel solutions to complex problems and tasks. Although classical statistical methods are capable of both correlation discovery and prediction, ML methods are better suited for identifying patterns, constructing features, and making predictions from large, complex, and heterogeneous data because they are usable and generalizable across a variety of data types and allow analysis and interpretation across complex variables. ML methods thus complement and extend existing statistical methods and can be used in highly innovative areas such as omics, radio-diagnostics, drug discovery, and personalized treatment. Of course, ML methods also have their limitations. The success of a ML project depends on the number of observations, the number of features, the selection and parameterization of the features as well as the quality of the underlying data and the chosen algorithm for the model $(90,91)$. $\mathrm{ML}$ also represents a component of AI research and development.
$\mathrm{AI}$ is a computer system that is able to integrate relevant information and make a rational and logical decision that leads to the best possible outcome.

ML is an important component of a modern DT in healthcare (92), that can be defined as a "virtual mirror of ourselves that allows us to simulate our personal medical history and state of health using data-driven analytical algorithms and theory-driven physical knowledge" (93) as well as to exploit the synergies resulting from their combination. That is, a DT uses the induction approach (statistical models that learn from data) and the deduction approach (mechanistic models that integrate multiscale knowledge and data) to provide accurate predictions of pathways to maintain or restore health (45). A DT consists of numerous dynamic and multidimensional parameters. Dynamic data means that the data from which the digital image of the patient is created are both historically available data and continuously updating and accumulating data from that person's life, e.g., data on the medical condition, data on the person's living environment, data on how a drug is tolerated or a therapy is accepted. The multidimensionality of the data arises from the many different sources from which the data come, such as monitoring data, data from the patient's social milieu, data from sensors, or clinical data. The dynamic and multidimensional nature of the data collected also distinguishes DT from other classical approaches such as clinical decision support systems (CDSS). A CDSS is used to make recommendations for appropriate tests and procedures from historical electronic health record (EHR) data using diagnosis of a condition and analysis of symptoms to help HCPs make informed decisions. The recommendation is the main component of a CDSS, which can be recorded in medical documents or coded in software as algorithms and rules $(94,95)$. However, the DT is not just a pure data collection approach for recommendations; it also correlates these data with each other and uses algorithms to incorporate the data meaningfully and purposefully into a simulation process with defined clinical (and economic) goals (95). The ability to simulate and model medical devices as well as pharmaceutical treatments on the computer enables faster and more cost-effective development than under real conditions $(45,48)$, without any risk for patients: "Making mistakes on computer models instead of people" (96).

The use of DTs in medical care is still in its infancy. So far, only in a few areas of medicine, DTs were applied, such as oncology (97-99), geriatrics $(100,101)$, cardiology $(45,102-$ 106), epidemic outbreaks (107), genomic medicine (48, 108), internal medicine $(109,110)$, orthopedics (111) and vascular medicine $(112,113)$. For example, Corral-Acero et al. present early steps of a DT in the field of cardiovascular medicine by describing the synergies between mechanistic and statistical models, the pillars of the DT (45). Topol describes "highperformance medicine" with the help of AI for HCPs in different disciplines like radiology, pathology, dermatology, ophthalmology, cardiology and gastroenterology (114) and gives an overview over selected reports of machine- and deep-learning algorithms to predict clinical outcomes and related parameters. Laaki et al. developed the prototype of a DT for real-time remote 
control of remote operations over mobile networks (81). Bruynseels et al. show how DTs are based on in-silico representations of an individual that dynamically reflect molecular status, physiological state and lifestyle over time (46).

Concrete implementations of digital twins can already be found for organs such as the heart, for example, by the French software company Dassault Systèmes (115) or by Siemens Healthineers in Germany (116). Siemens Healthineers has used data collected in a huge database of more than 250 million annotated images, reports and operational data. The AI-based DT model was trained to weave data about a heart's electrical properties, physical characteristics and structure into a 3D image. The technology was tested on 100 digital heart twins from patients treated for heart failure in a six-year study. Preliminary results of the comparison between the actual outcome and the predictions the computer made after analyzing DT status seemed promising. French startup Sim\&Cure developed a DT system that virtualizes a patient-based aneurysm and surrounding blood vessels. After a patient with aneurysm is prepared for surgery, a DT represented by a 3D model of the aneurysm and surrounding blood vessels is created by processing a 3D rotational angiography image. The personalized DT allows surgeons to perform simulations and helps them gain an accurate understanding of the interactive relationship between the implant and the aneurysm. In less than five minutes, numerous implants can be assessed to optimize the procedure. Preliminary studies have shown promising results (117).

\section{CONCEPT OF DIGITAL TWINS IN THE MANAGEMENT OF MULTIPLE SCLEROSIS}

Our vision is generating and implementing DTs in management of MS in order to improve diagnosis, treatment and management strategies as well as patient participation and compliance. DTs are a revolutionary tool for an improved characterization and prediction of disease course and for deep clinical phenotyping of pwMS (118). In this regard, big data analysis via ML supports visualization of the DTMS at different stages of MS and enables further therapeutic decisions. There are no elaborated DTs yet, but there are starting points and perspectives. For instance, Walsh et al. use an unsupervised ML model to learn the relationships between covariates commonly used to characterize subjects and their disease progression in clinical trials in MS (118). Recently, a research group from Sofia University in Bulgaria performed a first exercise of simulation of DTs. Petrova-Antonova et al. developed a web-based DT platform for MS diagnosis and rehabilitation that consists of two components: a transactional application that automates tests for MS diagnosis and rehabilitation, and an analytic application that provides data aggregation, enrichment, analysis, and visualization that can be used in any instance of the transactional application to generate new knowledge and support decision making. However, the analytical application is currently undeveloped and subject to further research (119).

We consider that, due to the complexity and long-term nature of MS, a particularly large and multidimensional amount of data must be collected and organized for the construction of DTMS.
These data must be of high quality, i.e. they must be collected correctly and represent the patient as accurately as possible. In addition to quality, a high quantity and frequency of data collection must also be achieved in the long term. To create DTMS and keep them updated with follow-up data, parameters related to the patients physiological status data (structured clinical data, para-clinical and multi-omics data, and patient-reported data) and to procedures (diagnostic workup, treatment, monitoring as integrated into personalized clinical pathways) should be collected, analyzed, visualized and correlated (Figure 1). The evolving and self-updating DTMS can be used simultaneously with ML algorithms to make smarter predictions and decisions as a learning health system (LHS) (120).

\section{Patients Physiological Status Data}

Patients' physiological status data content of DTMS includes structured clinical and para-clinical data, some of them as digital data, as well as multi-omics and patient reported data.

Structured clinical data are key parameters of deep clinical phenotyping and prerequisite for the data content of DTMS (30, 121). Taking the patient's history is traditionally the first important step in the evaluation of pwMS, which focuses on relapses and/or disease progression in the different neurological functional systems. Contextual parameters including lifestyle factors, comorbidities (122), psychological factors, emotions and sociodemographic factors (123-125) must also be recorded, assessed through the medical record and the conversation between physician and patient. There are attempts to standardize and quantitate MS relevant neurological history, such as e.g. the MSProDiscuss tool in the assessment of secondary disease progression (126). Further clinical evaluation e.g. by neurological examination is indispensable in MS for the quantitative measurement of the extent of the disorder, which is in turn required to find out how the disease is evolving and the influence the different forms of treatment are having on it. In recent years, the Expanded Disease Disability Scale (EDSS) has been an essential, irreplaceable scale in MS which has been improved in the past years by different approaches (127-129). However, other additional clinical instruments have been introduced to quantitate the different multidimensional aspects of MS as fatigue, cognition or walking function (130, 131). The Multiple Sclerosis Functional Composite (MSFC) provides a functional assessment of different key functions (upper and lower extremities, cognition) that is used more and more frequently in MS and has been proven to be highly sensitive in the evaluation of very important clinical trials. These complex data could allow clinical phenotyping of MS in terms of disease activity (132) or symptom-specific phenotypes (133). Because DTs are datadriven approaches, it is not advisable to assume that the same monitoring procedures already used by the clinician in everyday practice are sufficient to establish a model for comprehensive digital representation of pwMS. Therefore, a combination of different clinical outcome measures is highly recommended (134). Initiatives to standardize the collection of clinical data are on the way (135). 


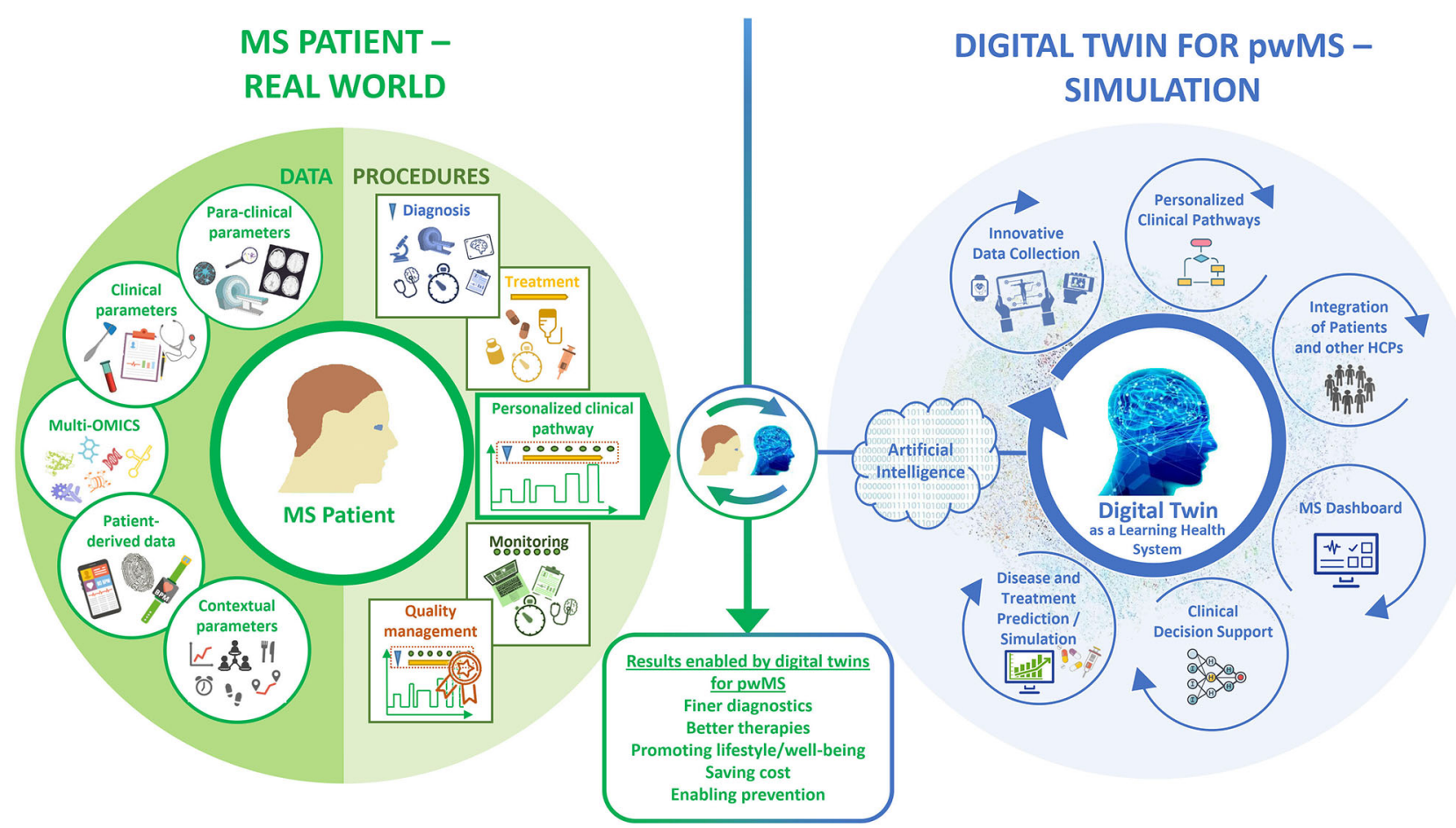

FIGURE 1 | Concept of a digital twin for pwMS.

Para-clinical data are of great importance for diagnosing, phenotyping and monitoring MS. Lab data ranging from standard laboratory to state of the art immunological or neurobiological parameters (136-139). Implementing standard lab data from clinical practice into a comprehensive approach of DTMS can complete the fundamental quest of real world evidence for individually improved treatment decisions and balanced therapeutic risk assessment $(140,141)$. As the MS disease process takes place in the CNS, analysis of cerebral spinal fluid is of high importance $(123,124)$. In addition to emerging immunological and neurobiological biomarkers, new technologies could be used for data collection for the DTMS as it has been described by Meyer zu Hörste (136) and will be described among multi-omics approaches. Neuronal destruction makers (e.g. neurofilament light chain) seem to be an excellent tool to measure subclinical MS disease activity in research and clinical studies $(125,142,143)$, but final validation and transfer in clinical practice would be optimal in the setting of the multidimensional approach of DTMS.

The importance of CNS imaging has steadily increased in recent years and is expected to continue to grow in light of new sequencing techniques and applications related to pathophysiology and prediction $(144,145)$. As a biophysical technique for measuring magnetic properties and generating weighted images of relative tissue contrasts, MRI offers both volumetric and dynamic quantitative means of detecting pathological tissue changes. These represent a promising approach to optimizing MS management through in vivo monitoring in the assessment of the course of chronic diseases by recording their disease-related dynamics or treatment-induced effects $(146,147)$.
To implement imaging into DTMS, it is essential to standardize MRI acquisition $(148,149)$. The aim of this approach is to increase the sensitivity of MRI analysis to the smallest disease-related tissue changes. The acquisition of 3D-resolved sequences is important, as these, on the one hand, allow the free exploration of the image data by reformatting and, on the other hand, allow an optimal adaptation to the preliminary examination through modern $3 \mathrm{D}$ registration. In addition, only these 3D-resolved sequences form the basis for computer-assisted image data analysis and volumetric measurements, which should further increase precision in the future. Recent advances of CNS imaging could be probably transferred more easily into clinical practice by their integration into DTMS. Using this platform to put imaging data in context with other multidimensional data offers unique possibilities of validation and implementation. Thus, in future, quantitative MRI will enable a detailed characterization of brain tissue by generating a large number of numerical results (150). More than a thousand parameters can be generated if a detailed segmentation of the brain is considered, making group studies complex and inefficient by parametric techniques of data analysis (150). The large volume of MRI data can only be approached by AI, an essential tool of the DTMS (151). Finally, by measuring both volumetric and dynamic quantitative means (lesions and atrophy), different MRI phenotypes of individual patients can be described by MRI-categorization (152) which could be an important component of DTMS. In addition to MRI, data obtained through other imaging biomarkers such as OCT (153) or Positron emission tomography (154) can be used as well.

Digital phenotyping. Several clinical and para-clinical data can be collected digitally (digital phenotyping with digital 
biomarkers). Digital biomarkers are measures to collect objective data on biological (e.g., blood glucose, serum sodium), anatomical (e.g., mole size), or physiological (e.g., heart rate, blood pressure) parameter with the use of a biosensor (portable e.g. smartphones, wearable, and implantable devices), followed by the use of algorithms to transform these data into interpretable outcome measures (155-157). They are used for assessing e.g. cognitive function (158) or fatigue (159).

Sensor-based, portable measurement systems can be used both in the clinical setting and in the patients' individual everyday life (at home). In the clinical setting functional tests and gait analysis can be performed digitally. The Multiple Sclerosis Performance Test (MSPT) is a digital adaptation of the MSFC with additional elements added $(160,161)$ and measures health status via iPad with questionnaire on health status, processing speed with Processing Speed Test (PST) (162), manual skills with 9-Hole-Peg-Test (9-HPT) and walking speed with Timed 25-Foot-Walk (T25-FW) (160). Multidimensional gait analysis can be performed with measurement of walking speed (T25-FW), measurement of endurance [2-Minute Walk Test, 2MWT $(163,164)]$ and measurement of balance and gait quality on a sensor-based walking mat (GAITRite ${ }^{\circledR}$-System, Mobility Lab-System) (131). For the digital measurement of data in patient-specific everyday life (at home) there are various patient apps such as Floodlight, diverse fitness tracker and health apps available $(165,166)$. They make it possible to collect realistic data relevant to everyday life via remote sensing in addition to the regular medical consultations. Thus, a more comprehensive insight into the patients' daily life as well as a more closely meshed progression monitoring is made possible. Clinical and para-clinical data (including lab and imaging data) are more and more collected in digital format and a standardized way which is an important step for integration in DTMS. A key role in the development of global standards of data related to patients or health cases is played by various organizations such as the Clinical Data Interchange Standards Consortium (CDISC), the Critical Path Institute (C-Path), and the Health Level 7 organizations (167). In clinical care, the development of digital neurological assessment tools such as Neurostatus-eEDSS and tablet-based MSPT, as well as real-time 3D motion capture systems for recording motor dysfunction in MS patients, play the most important role. The MS Data Alliance has already developed digital tools for aggregating, harmonizing, and sharing real-world data from multiple sources by creating a common data model. EHR also play a critical role in standardized and accurate digital documentation of clinical data, and several of these already exist, such as the MS BRIDGE, RC2NB, MSDS3D and MSBase EHR systems (168).

Multi-omics as innovative approach will have to be a part of the DTMS as well especially to increase knowledge about MS (169-171). The complex and dynamic processes in the neurobiological and immune networks are of significant importance in MS as in other chronic diseases. Advances in high-throughput "omics" technologies (e.g., genome, transcriptome, proteome, epigenome, metabolome) are enabling MS care to move from a "one-size-fits-all" toward a personalized approach analyzing the correlation of multi-omics with the clinical and para-clinical phenotypes of the individual MS patient (Figure 2). Multi-omics approaches involving large populations of pwMS and interrogating millions of markers with similar biochemical properties can help to elucidate the molecular mechanisms underlying MS and provide both potential biomarkers and pharmacological targets for a more detailed patient stratification and personalized treatments (172). Genomic and proteomic studies have sought to understand the molecular basis of MS and find biomarker candidates. Regarding genomic and proteomic studies, advances in next-generation sequencing and massspectrometry techniques have been of great importance to generate an unprecedented amount of relevant data (173). In order to study complex biological processes holistically, it is imperative to adopt an integrative approach. Multi-omics data should be combined to shed light on the interrelationships of the biomolecules involved and their functions. With the rinsing of high-throughput techniques and the availability of multi-omics data from a large number of samples, promising tools and methods for data integration and interpretation have been developed (174). In the field of MS, this strategy was successful for the development of novel data science techniques for exploring these large datasets to identify biologically relevant relationships and ultimately point towards useful biomarkers which have been discovered in recent years $(124,173)$.

Patient-reported data like questionnaire data complement the clinical data and complete the picture of the DTMS by including the patients' perspective of their disease. They are divided into patient reported outcomes (PRO) and patient reported experiences (PRE). PRO is an umbrella term for health outcomes that are directly and subjectively reported by patients without interpretation of the patients' response by a clinician or anyone else $(175,176)$. PRO are measured for outcomes like quality of life by the Quality of Life in Neurological Disorders $(177,178)$, and like walking and mobility skills by the Twelve Item MS Walking Scale (164, 179) or the Early Mobility Impairment Questionnaire (180). PRE measure "patient's perception of their personal experience of the healthcare they have received" (181). PRE measures assess patients' perception of their experience of the received healthcare collected through questionnaires (182). Efforts to standardize data are already underway. The PROMS (Patient Reported Outcomes for MS) initiative aims to identify PROs, including actively and passively delivered digital performance measures, to standardize outcomes in both research and clinical decision making (183).

Thus, model building for a DTMS already requires a comprehensive set of monitoring tools to be tested on a representative sample. To a certain extent, this also describes the scope of the instruments, which must later be applied to individual patients in practice in order to derive a comparable trajectories.

\section{Procedures}

An optimal management of pwMS requires the performance of certain procedures as e.g. assessments of clinical and para- 

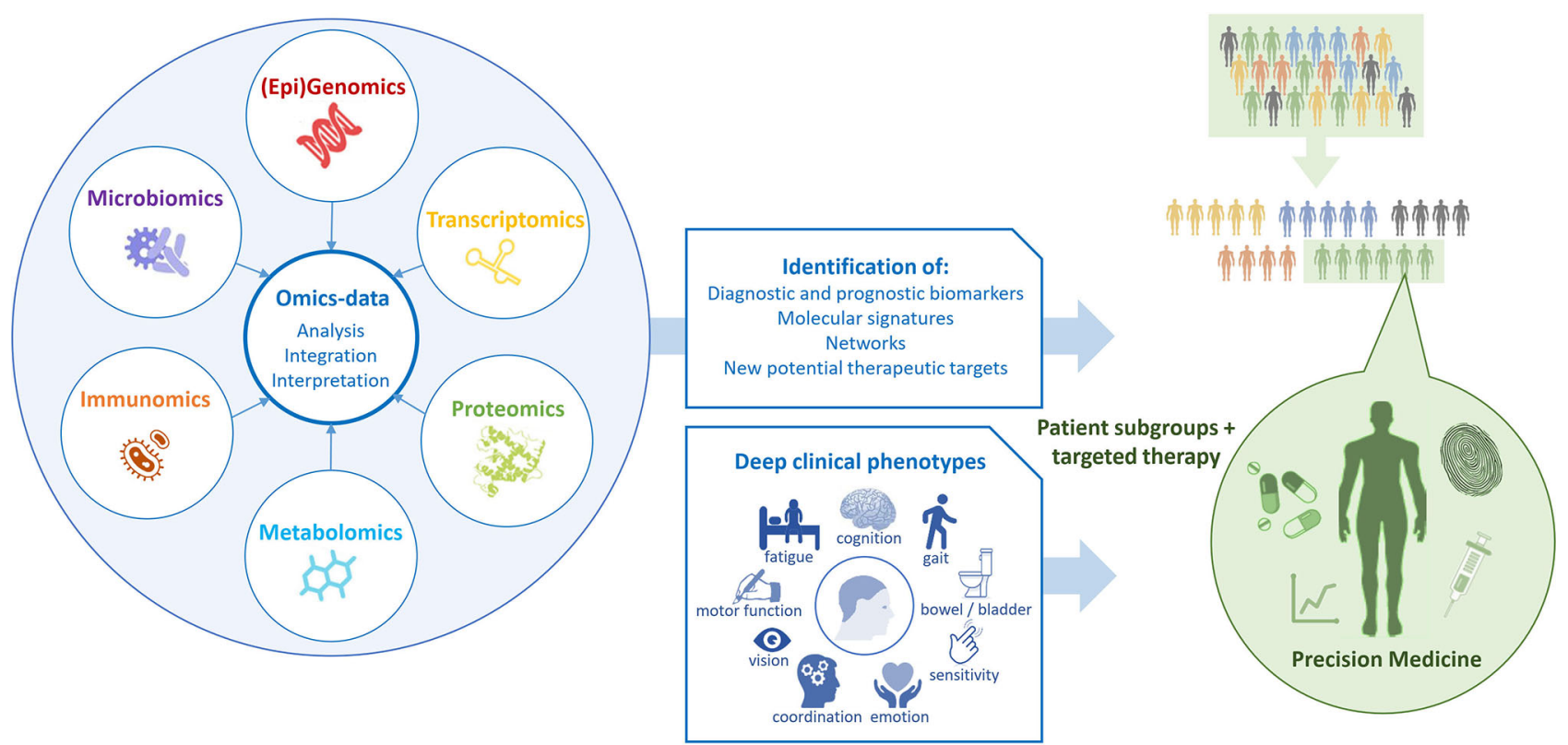

FIGURE 2 | Multi-omics for precision medicine.

clinical parameters at high quality and at defined time points. In addition to the more general and non-concrete guidelines related to standard clinical practice, the Brain Health Initiative has provided for the first time specific "core," "achievable," and "aspirational" time frames for individual treatment steps in diagnosis, treatment, and monitoring (14). Achieving these standards of MS management in the individual patient to increase quality of care for pwMS will be facilitated by integrating such procedural components of these clinical pathways into the DTMS.

Diagnosis of MS is based on defined diagnostic criteria [McDonald criteria (5)] and relies on various examination methods (184), none of which alone is capable of making the diagnosis of MS as the differential diagnosis is quite complex. The procedural component of diagnostic workup in DTMS will assist in collecting data in optimal time considering type and stage of disease, pertinent symptoms and comorbidities, time between the first referral to the neurologist and MRI, etc.

Treatment. The therapeutic management in MS includes DMTs, treatment of acute relapses, and symptomatic therapies, which are usually combined and individually adapted. In particular, the history and the stage of the disease, degree of disability, the primary symptomatology, form and dynamics of the course of the disease, age, gender and desire to have children, concomitant and previous diseases, concomitant and premedication as well as the individual life situation of the patient must be taken into account. The DTMS will assist in the selection and monitoring of individual treatments. In order to assess possible adverse events and reactions, individual treatments need a defined treatment-related clinical pathway including clinical and para-clinical assessments, which have to be integrated into the DTMS.
Monitoring. An optimal primary goal of MS therapy should be the achievement of no evidence of disease activity (NEDA) (9, 185). Specifically, this means the absence of relapses, new or enlarged lesions on MRI, clinical disability progression and loss of brain volume (=NEDA-4). The NEDA status has to be assessed by procedures of MS monitoring to detect disease progression and relapse as well as the monitoring of disease activity and symptoms. The importance of frequent high quality monitoring in routine clinical management of MS is pointed out by numerous authors with reference to various studies and the comprehensive data on the significance of relapses, early EDSS changes, and the role of MRI (186-188). As monitoring of MS is a lifelong challenge for patients and HCPs, its integration into DTMS will assist in keeping up this essential long term assessment.

Personalized clinical pathways that integrate these procedures are also included in the design of DTMS and should be available for the HCP and patient together to ensure the best possible outcome.

\section{Construction of Digital Twins for Multiple Sclerosis}

Prediction models based on statistical models already exist. For example, Stühler et al. and Kalincik et al. have investigated the individual response of pwMS to disease-modifying therapies using generalized linear models. However, in both studies, data density and quality were insufficient because, among other reasons, the cohorts were too small or there were data gaps in MRI data or data could not be comprehensively included (189191). With the DTMS, all historically and currently available data should be continuously included in the analysis, if possible, to increase predictive power. In addition to the standardized and 
digitized parameters on patients' physiological status data and procedures, the available prior knowledge in the field of MS should also be included in the construction of the DTMS. In addition to existing guidelines (14), this also includes further expert knowledge from the practice of clinical care of pwMS as well as possible knowledge about factors that can positively or negatively influence the disease, e.g. comorbidities, nutrition, physical activity and cessation of smoking.

Before the DTMS is implemented in practice, it is essential to check which data are absolutely necessary to collect and how the data collection can be done in such a way that it burdens the patient and HCP as little as possible. This is also important from an economic point of view, as the collection of all the abovementioned data types is associated with high costs. This examination could be done by different tools. Basically, a targeted literature review on parameters particularly frequently used for prognostic purposes would be necessary, which could be complemented by a survey among experts. Since the strength of ML methods lies in discovering hidden patterns, test runs of the DTMS with the integration of different parameters (classes) would be conceivable, the results of which would be tested in a representative sample. Some work already provides clues in this regard. As Pinto et al. have pointed out in their work on prediction of MS progression using ML methods, relevant clinical information may include EDSS, functional systems and CNS functions affected during relapses, as well as age and gender (192). In any case, data acquisition should be done digitally and in an automated manner, if at all possible, with a view to minimizing patient disruption. There is a need for further research in this area which data have been collected from patient and HCP.

\section{USE CASES IN CARE OF MULTIPLE SCLEROSIS}

DTMS perform a new kind of deep phenotyping by processing all data and procedural content in its complexity with innovative tools. Taking into account all previously defined medical and contextual parameters, which are very closely interwoven with the patient and his identity, the DTMS provides decision templates based on calculated probabilities. HCPs, patients, and all those involved in their care, have therefore an individualized roadmap of which examinations, tests, and therapies to pursue in the near future. In this process, the DTMS controls and monitors the entire disease management process and can correct any deviations. Thus, the DTMS is also a tool for measuring the process quality of a treatment. This results in a number of application scenarios that will fundamentally improve management of MS (Figure 1).

\section{Innovative Data Collection}

For linking large amounts of data from different sources, suitable interfaces and modular database systems should be available that can integrate different external systems. The ability of different systems to work together is called interoperability. To achieve interoperability and also flexibility, the use of an interoperability standard, such as HL7 FHIR (193), and standard interfaces, e.g. IHE XDS.b for Germany (194), should be ensured (195). This is where a MS portal such as the Integrated Care Portal Multiple Sclerosis (IBMS) (195) could be used, to which both patients and HCPs can contribute different types of data. Patient data collected via apps or questionnaires flow into the patient portal, which is part of a management system for MS. The $\mathrm{HCP}$, in turn, can see this data in the system and enter content related to the data and processes there. In the further course, data enter the database continuously, which can be used for the DT.

\section{Clinical Pathways}

Clinical pathways are particularly suitable for the seamless care of chronically ill patients across various health sectors. They describe the entire path of patients during care (the "patient journey") and unite the multidisciplinary setting, the local conditions and the current state of evidence research (195). Clinical pathways define goals and milestones of care and support shared decision making between HCPs and patients by also providing patients with a picture of their stage of disease (30, 195-198).

As intelligent systems, DTs traverse the clinical pathway, serving as a guide for HCPs and patients through treatment with an individual roadmap. Integrated into clinical management systems, clinical pathways can thus also serve as quality assurance tools for HCPs and patients. In this way, patients can actively participate in the quality improvement of their treatment process. HCPs, in turn, have the opportunity to optimize treatment steps based on specific quality indicators. These quality indicators are derived from existing MS guidelines and consensus standards [e.g., the International Brain Health Initiative consensus standards (14)]. On the one hand, they address temporal concerns for diagnosis, treatment, and monitoring phases, e.g., the maximum time between initial presentation and the acquisition of an MRI. On the other hand, quality instruments are integrated to measure the assurance of desired outcomes for pwMS, e.g., whether patients who have mobility or fatigue issues are offered support (199) or whether patients experience coordinated care with clear and accurate information exchange (200). Defining and measuring quality indicators is the goal of the currently running project "Path-based Quality Management in MS Care" (QPATH4MS) at the MS Center Dresden (Germany).

\section{MS Dashboard for Visualization}

Visualization helps to present complex data in an understandable and clear way. The so-called MS dashboard visualizes high-dimensional disease characteristics and individual clinical pathways. The HCP can present the possibilities played out by means of the DT to the patient to discuss therapy options and clinical pathways with the patient. Through an adaptive display, it is possible to present the individual patient pathway, therapy options, treatment alternatives and the associated risks and challenges in a simplified form for the patient as a layperson and for the HCP as an expert. Within this framework, HCPs and patients can determine the ideal therapy and management of MS through 
shared decision-making. Thanks to the visualized simulation of the DT, the HCP has time to address all patients' questions and concerns in detail. Examples of existing dashboards for displaying individual patient data at a glance include the walking assessment dashboard as part of the multidimensional digital patient management system $\operatorname{MSDS}^{3 \mathrm{D}}$ (201, 202), showing the results of clinical multidimensional walking assessment and daily smart monitoring longitudinally (131), and the MS BioScreen, that integrates multiple dimensions of disease information: clinical evolution, therapeutic interventions, brain, eye, and spinal cord imaging, environmental exposures, genomics, and biomarker data $(56,203)$.

\section{Integration of Patients and Other Healthcare Professionals}

The visualization of the complex data involved in the medical and therapeutic decisions may foster the communication between HCPs and patients. This would support the involvement of patients in healthcare decisions and management of their disease. In this way, DTs also serve as a shared decision-making tool for HCPs and patients, who will play a much more active role in their own healthcare management in the future. For example, this could empower the patient to become an active member of the MS management team, from providing data (including data from biosensors, for example) to recording/tracking notable events and daily care to prognostic tools. As a result, a much more granular, continuous perspective on MS and its progression is provided, which would be more complete than traditional (brief and irregular) clinical assessments.

\section{Clinical Decision Support System}

A DT also acts as clinical decision support system (CDSS) that supports HCPs in clinical decision making by providing evidence-based medical knowledge and patient-related information $(204,205)$. The goal is to enable the HCP to make the best possible clinical decision for the patient, with the best possible chance of a positive outcome. CDSSs are often supported by ML-based algorithms. The ambiguous patterns of MS (e.g., in etiology, progression, clinical presentation, and response to drug therapies) make $\mathrm{ML}$ algorithms optimal tools to automate the detection of patterns and regularities in MS data. CDSSs are very beneficial in the context of MS, but are not yet well established. There is an increasing need for CDSSs in MS to help HCPs make the right decision among multiple alternatives in time (206).

\section{Simulation and Prediction of Disease and Treatment Outcomes}

Modeling the course of MS, especially predicting progression, is challenging due to the complexity of the outcomes and its varying course. The DT offers the possibility of predicting several probable disease courses and provides models for estimating possible treatment effects for individual patients. Taking into consideration all of a patient's individual parameters, potential side effects, costs incurred, and individual circumstances and patient satisfaction, the DT can suggest the option with the highest benefit for the patient. There are initial approaches to predicting disease progression using ML. For instance, Pinto et al. used clinical information to develop a ML system to explore the disease evolution in pwMS in terms of conversion from RRMS to SPMS. EDSS score, majority of functional systems, affected functions during relapses, and age at onset were described as the most predictive features (192). Zhao et al. found that support vector machines incorporating short-term clinical and brain MRI data were better at predicting disease progression of MS and selecting patients for more aggressive treatments than logistic regression methods (207). Later, Zhao et al. compared common ML algorithms and so-called ensemble learning approaches. The latter were more effective and robust compared with single algorithms and offered increased accuracy for predicting disease progression of MS. Of the variables evaluated, EDSS, pyramidal function, and ambulation index were the most common predictors in predicting MS disease progression (208). Another study suggested that the concentration of serum cytokines could be used as prognostic marker for the prediction of MS (209). Datadriven subtyping and staging of MS could better predict subsequent clinical course and response to treatment compared with clinical classification or baseline EDSS. Data-driven subtyping has the potential to prospectively improve patient outcomes.

DTs help to understand disease's dynamics and thus, advise HCPs on medication intake. With regard to drugs, it is quite conceivable that in the future clinical trials will also be conducted only with the help of DTs and no longer with the patients themselves.

From all that is known so far, the DT is a Learning Health System (LHS). LHS fuse healthcare delivery with research, data science, and quality improvement processes. The LHS cycle begins and ends with HCP-patient interactions and strives for continuous improvement in healthcare quality, outcomes, and efficiency (210). Based on the constantly new data collected through continuous monitoring and provided by the patient from the real world, the DT generates new knowledge, which in turn flows into the patient's further treatment, which is thus continuously improved. The parameter data continuously flow into the calculations of the DT - with each piece of information, the phenotype can be described more precisely. The therapy can thus be continuously adapted to the patient's disease state and life circumstances.

\section{CHALLENGES OF DIGITAL TWINS IN HEALTH CARE}

The use of DTMS promises to improve clinical decision making for individual patients, enhance patient communication, and improve quality of care. However, no uniform methods, standards, or norms yet exist for the development of DTs, and many challenges remain to unleash the potential of DTs $(49,66)$.

\section{Data Quality, Data Management and Algorithm Design}

Poor or missing data and information can lead to improper models and incorrect recommendations (trash in, trash out). In order for the DT to be statistically indistinguishable from its realworld counterpart, the data on which the DT is based must be of 
high quality and represent the patient as completely as possible $(118,120)$. Data quality in the broader sense also includes the standardized collection or standardization of data to ensure their reliability and to enable longitudinal and cross-sectional comparisons of data. In this context, data should preferably be carried out in digital form or at least recorded digitally instead of in paper form in order to facilitate standardization and thus comparability. There is currently no generally accepted, standardized scheme for the collection, documentation, and evaluation of data in MS, although recommendations and guidelines from various expert groups exist, which have already been described in the sections on patients physiological status data and procedures (135). For the purpose of generating a sufficiently large amount of data describing pwMS in a standardized multidimensional manner, many years of multicenter data acquisition are required. Only on this basis is it possible to create the necessary "critical mass" of data in the required density to enable long-term estimation of therapeutic outcomes. In addition, multidimensional and unstructured large data sets must first be structured and then integrated into meaningful algorithms before meaningful models can be created $(45,95,114)$. It should also be noted that the results of ML algorithms are usually based on a large number of parameters and criteria that can no longer be reproduced or fully understood by humans (135). Even if the models produce solid predictions, it may be impossible to deduce why they make good prediction.

\section{Data Privacy and Data Security}

Before DTs are created, it is essential to clarify who owns which data at what point in time and for how long, who has access to it under which conditions and for how long, who actually owns the "end product" of the DT, and who can use it and under which conditions. It is imperative that suitable governance structures be created for this purpose. Furthermore, data security is very important to avoid data gaps that could potentially be used for hacker attacks to the detriment of patients. It is also necessary to ensure the protection of privacy, which becomes more and more difficult with the increasing functionality of techniques. Patients must also be confident that their data is secure, transparent and accessible to them. Otherwise, the collection of patient data could increase mistrust rather than confidence in health systems. Simply providing technological advances is not enough, it is also necessary to ensure that it serves to improve well-being. Therefore, data privacy and transparency of data use must be respected with the full consent of patients. Informed consent should explicitly state the purposes for which the data collected from patients will be used $(49,93,120,211)$.

\section{Ethical Concerns}

DT models could exaggerate racial and other bias $(46,212)$ and could lead to or reinforce inequalities in health care (46): if a group is misrepresented in the data used to create models, this group may receive suboptimal treatment (213). An example shows that a computer model classifies patients with a history of asthma who have pneumonia as patients with a lower risk of mortality than those who have pneumonia only. However, the context was completely ignored, namely that this is an artifact of clinicians admitting and treating such asthma patients earlier and more aggressively (212). Another important ethical issue related to predicting the course of disease is whether and in what way the prognosis should be communicated to patients. How does a patient deal with the knowledge that, according to the prognosis, he or she will soon be in a wheelchair, for example? Do patients have a right to "not know"? In addition, the extent to which patients will be able to decide autonomously what is good or bad for them, and to what extent this will be determined by the algorithms that claim to propose the most optimal solution based on the available data, needs to be reconsidered. In this context, "dataism" could become a new form of medical paternalism. Patients must therefore develop an appropriate relationship with their personal DT and develop the ability to make informed decisions in the face of strong data-driven personalized models (46).

\section{Individual Concerns and Trust in Applications of Al}

The role of humans or users of AI applications should not be underestimated, and trust is a crucial factor in this context (120, 214). The fear of new not-yet-established technologies like AI is a barrier to trust (120). HCPs may not trust the decisions of machines if they do not understand the involved algorithms. Additionally, HCPs could experience fear of being replaced by machines. However, AI will not replace the HCP (114), but will support and provide more time for consultation with the patient - one of the crucial aspects of medical care (215). Decisions based on AI can help the HCP make good decisions, if they "keep human intelligence up to date and take into account the social, clinical and personal context" (212). In the case that the DT's recommendations contradict his or her own, the HCP must dispose of an action plan for further decision-taking. Otherwise, more data can contribute to the uncertainty of the medical thinking.

In order to establish the concept of the DT despite all the challenges mentioned, guidelines, gold standards, benchmark tests and governmental legislation, as has been achieved in Estonia, are therefore necessary $(45,114)$. Before using DTs in patient care, it is imperative that targeted studies, publication of results in peer-reviewed journals and clinical validation in a realworld environment are carried out (114). Nevertheless, HCP should proactively guide, supervise and monitor the introduction of DTs as partners in patient care (212).

\section{DISCUSSION}

With the development of a DTMS, it is possible to improve clinical decision-making for individual patients, patient communication, shared decision-making, and thus quality of care. Before DTs can be used in patient care, they must be validated by studies and experts, as well as by real-world investigations to show the effectiveness and safety of their methods. In addition, there are still a number of challenges to overcome on the road to using DTs, such as ensuring data security and privacy and the accuracy of the data on which the 


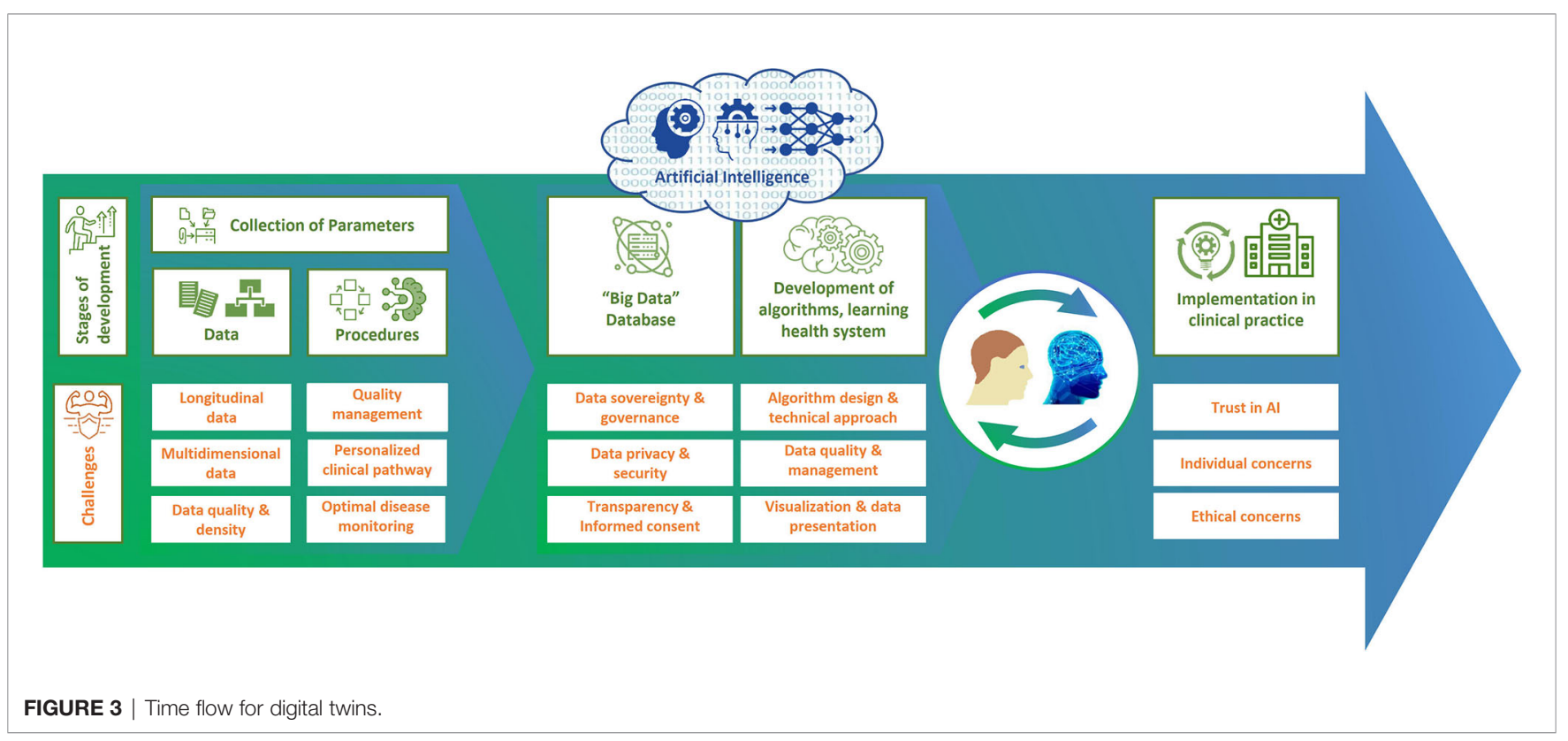

DT is based (Figure 3). It should also not be underestimated that the development of a DTMS is very complex and therefore expensive and may also increase the complexity of monitoring in clinical practice. Therefore, further research should be included in the development to inform which data contribute most to predictability, how this predictability can be assessed, and how this approach can be feasibly and cost-effectively integrated into health care. Further work will also be required to see whether and how predictive models can be constructed. However, a basic DTMS can serve as a starting point that will grow and evolve over time. During this process, the HCP should proactively guide, oversee, and monitor the introduction of DTMS as partners in patient care. By analyzing all possible factors of MS, DTMS will help make precision medicine and patient-centered care a reality in everyday life. This will ultimately refine diagnostics and monitoring, improve

\section{REFERENCES}

1. Filippi M, Rocca MA, Ciccarelli O, De Stefano N, Evangelou N, Kappos L, et al. MRI Criteria for the Diagnosis of Multiple Sclerosis: MAGNIMS Consensus Guidelines. Lancet Neurol (2016) 15(3):292-303. doi: 10.1016/ S1474-4422(15)00393-2

2. Goldenberg MM. Multiple Sclerosis Review. P T Peer-Rev J Formulary Manage (2012) 37(3):175-84.

3. Huang WJ, Chen WW, Zhang X. Multiple Sclerosis: Pathology, Diagnosis and Treatments (Review). Exp Ther Med (2017) 13(6):3163-6. doi: 10.3892/ etm. 2017.4410

4. Khurana V, Sharma H, Medin J. Estimated Prevalence of Secondary Progressive Multiple Sclerosis in the USA and Europe: Results From a Systematic Literature Search (P2.380). Neurology (2018) 90(15 Supplement): P2.380.

5. Thompson AJ, Banwell BL, Barkhof F, Carroll WM, Coetzee T, Comi G, et al. Diagnosis of Multiple Sclerosis: 2017 Revisions of the McDonald Criteria. Lancet Neurol (2018) 17(2):162-73. doi: 10.1016/S1474-4422(17)30470-2

6. Weinshenker BG. The Natural History of Multiple Sclerosis: Update 1998. Semin Neurol (1998) 18(3):301-7. doi: 10.1055/s-2008-1040881 therapies and patient well-being, save economic costs, enable prevention, expand treatment options and empower patients.

\section{AUTHOR CONTRIBUTIONS}

IV and TZ designed and conceptualized paper as well as revised the manuscript for intellectual content. $\mathrm{HI}, \mathrm{AD}, \mathrm{RH}$ and $\mathrm{AD}$ revised the manuscript for intellectual content. All authors contributed to the article and approved the submitted version.

\section{ACKNOWLEDGMENTS}

We are grateful that the IBMS and QPATH4MS projects were funded by the ERDF.

7. Lublin FD, Baier M, Cutter G. Effect of Relapses on Development of Residual Deficit in Multiple Sclerosis. Neurology (2003) 61(11):1528-32. doi: 10.1212/01.WNL.0000096175.39831.21

8. Thompson AJ, Montalban X, Barkhof F, Brochet B, Filippi M, Miller DH, et al. Diagnostic Criteria for Primary Progressive Multiple Sclerosis: A Position Paper. Ann Neurol (2000) 47(6):831-5. doi: 10.1002/1531-8249 (200006) 47:6<831::AID-ANA21 >3.0.CO;2-H

9. Weinstock-Guttman B, Medin J, Khan N, Korn JR, Lathi E, Silversteen J, et al. Assessing 'No Evidence of Disease Activity' Status in Patients With Relapsing-Remitting Multiple Sclerosis Receiving Fingolimod in Routine Clinical Practice: A Retrospective Analysis of the Multiple Sclerosis Clinical and Magnetic Resonance Imaging Outcomes in the USA (Ms-Mrius) Study. CNS Drugs (2018) 32(1):75-84. doi: 10.1007/s40263-017-0482-4

10. Ziemssen T, Derfuss T, de Stefano N, Giovannoni G, Palavra F, Tomic D, et al. Optimizing Treatment Success in Multiple Sclerosis. J Neurol (2016) 263(6):1053-65. doi: 10.1007/s00415-015-7986-y

11. Inojosa H, Proschmann U, Akgun K, Ziemssen T. A Focus on Secondary Progressive Multiple Sclerosis (SPMS): Challenges in Diagnosis and Definition. J Neurol (2021) 268(4):1210-21. doi: 10.1007/s00415-01909489-5 
12. Inojosa H, Proschmann U, Akgün K, Ziemssen T. Should We Use Clinical Tools to Identify Disease Progression? Front Neurol (2021) 11:628542-. doi: 10.3389/fneur.2020.628542

13. Montalban X, Gold R, Thompson AJ, Otero-Romero S, Amato MP, Chandraratna D, et al. Ectrims/Ean Guideline on the Pharmacological Treatment of People With Multiple Sclerosis. Mult Scler (2018) 24(2):96120. doi: $10.1177 / 1352458517751049$

14. Hobart J, Bowen A, Pepper G, Crofts H, Eberhard L, Berger T, et al. International Consensus on Quality Standards for Brain Health-Focused Care in Multiple Sclerosis. Mult Scler (2018) 25(13):1809-18. doi: 10.1177/ 1352458518809326

15. Reich DS, Lucchinetti CF, Calabresi PA. Multiple Sclerosis. New Engl J Med (2018) 378:169-80. doi: 10.1056/NEJMra1401483

16. Yaldizli Ö, Kappos L. Klinische Grundlagen Der Multiplen Sklerose. S Egli, editor. Berlin and New York: Springer (2011).

17. Kip M, Schönfelder T, Bleß H. Versorgungssituation in Deutschland. In: Weißbuch Multiple Sklerose Berlin: Springer (2016).

18. Alkhawajah M, Oger J. When to Initiate Disease-Modifying Drugs for Relapsing Remitting Multiple Sclerosis in Adults? Multiple Sclerosis Int (2011) 2011:724871. doi: 10.1155/2011/724871

19. Merkel B, Butzkueven H, Traboulsee AL, Havrdova E, Kalincik T. Timing of High-Efficacy Therapy in Relapsing-Remitting Multiple Sclerosis: A Systematic Review. Autoimmun Rev (2017) 16(6):658-65. doi: 10.1016/j.autrev.2017.04.010

20. Ziemssen T. Dem MS-Phänotyp Auf Der Spur. DNP Der Neurologe Psychiater (2019) 20(5):33-6. doi: 10.1007/s15202-019-2277-6

21. Ziemssen T, Medin J, Couto CA, Mitchell CR. Multiple Sclerosis in the Real World: A Systematic Review of Fingolimod as a Case Study. Autoimmun Rev (2017) 16(4):355-76. doi: 10.1016/j.autrev.2017.02.007

22. Tacchella A, Romano S, Ferraldeschi M, Salvetti M, Zaccaria A, Crisanti A, et al. Collaboration Between a Human Group and Artificial Intelligence can Improve Prediction of Multiple Sclerosis Course: A Proof-of-Principle Study. F1000Research (2017) 6:2172. doi: 10.12688/ f1000research.13114.1

23. Marziniak M, Ghorab K, Kozubski W, Pfleger C, Sousa L, Vernon K, et al. Variations in Multiple Sclerosis Practice Within Europe - Is it Time for a New Treatment Guideline? Mult Scler Relat Disord (2016) 8:35-44. doi: 10.1016/j.msard.2016.04.004

24. Ohlmeier C, Gothe H, Haas J, Osowski U, Weinhold C, Blauwitz S, et al. Epidemiology, Characteristics and Treatment of Patients With Relapsing Remitting Multiple Sclerosis and Incidence of High Disease Activity: Real World Evidence Based on German Claims Data. PloS One (2020) 15(5): e0231846. doi: 10.1371/journal.pone.0231846

25. Ziemssen T, Thomas K. Treatment Optimization in Multiple Sclerosis: How do We Apply Emerging Evidence? Expert Rev Clin Immunol (2017) 13 (6):509-11. doi: 10.1080/1744666X.2017.1292135

26. Sellner J, Rommer PS. Immunological Consequences of "Immune Reconstitution Therapy" in Multiple Sclerosis: A Systematic Review. Autoimmun Rev (2020) 19(4):102492. doi: 10.1016/j.autrev.2020.102492

27. Sellner J, Rommer PS. A Review of the Evidence for a Natalizumab Exit Strategy for Patients With Multiple Sclerosis. Autoimmun Rev (2019) 18 (3):255-61. doi: 10.1016/j.autrev.2018.09.012

28. D'Amico E, Zanghì A, Gastaldi M, Patti F, Zappia M, Franciotta D. Placing CD20-Targeted B Cell Depletion in Multiple Sclerosis Therapeutic Scenario: Present and Future Perspectives. Autoimmun Rev (2019) 18(7):665-72. doi: 10.1016/j.autrev.2019.05.003

29. Montes Diaz G, Hupperts R, Fraussen J, Somers V. Dimethyl Fumarate Treatment in Multiple Sclerosis: Recent Advances in Clinical and Immunological Studies. Autoimmun Rev (2018) 17(12):1240-50. doi: 10.1016/j.autrev.2018.07.001

30. Ziemssen T, Kern R, Thomas K. Multiple Sclerosis: Clinical Profiling and Data Collection as Prerequisite for Personalized Medicine Approach. BMC Neurol (2016) 16:124. doi: 10.1186/s12883-016-0639-7

31. Abrahams E. Right Drug-Right Patient-Right Time: Personalized Medicine Coalition. Clin Trans Sci (2008) 1(1):11-2 doi: 10.1111/j.17528062.2008.00003.x

32. Jameson JL, Longo DL. Precision Medicine-Personalized, Problematic, and Promising. N Engl J Med (2015) 372(23):2229-34. doi: 10.1056/ NEJMsb1503104
33. National Research Council Committee on AFfDaNToD. The National Academies Collection: Reports Funded by National Institutes of Health. In: Toward Precision Medicine: Building a Knowledge Network for Biomedical Research and a New Taxonomy of Disease. Washington (DC: National Academies Press (US) Copyright @ (2011, National Academy of Sciences (2011).

34. Sugeir S, Naylor S. Critical Care and Personalized or Precision Medicine: Who Needs Whom? J Crit Care (2018) 43:401-5. doi: 10.1016/ j.jcrc.2017.11.026

35. Abrahams E, Ginsburg GS, Silver M. The Personalized Medicine Coalition. Am J Pharmacogenom (2005) 5(6):345-55. doi: 10.2165/00129785200505060-00002

36. Toward Precision Medicine. Building a Knowledge Network for Biomedical Research and a New Taxonomy of Disease. NR Council, editor. Washington, DC: The National Academic Press (2011). p. 142.

37. Ashley EA. The Precision Medicine Initiative: A New National Effort. JAMA (2015) 313(21):2119-20. doi: 10.1001/jama.2015.3595

38. Collins FS, Varmus H. A New Initiative on Precision Medicine. New Engl J Med (2015) 372(9):793-5. doi: 10.1056/NEJMp1500523

39. Collins H, Calvo S, Greenberg K, Forman Neall L, Morrison S. Information Needs in the Precision Medicine Era: How Genetics Home Reference can Help. Interactive J Med Res (2016) 5(2):e13-e. doi: 10.2196/ijmr.5199

40. Conrad K, Shoenfeld Y, Fritzler MJ. Precision Health: A Pragmatic Approach to Understanding and Addressing Key Factors in Autoimmune Diseases. Autoimmun Rev (2020) 19(5):102508. doi: 10.1016/ j.autrev.2020.102508

41. Jain KK. Textbook of Personalized Medicine. Switzerland: Springer, Cham (2021).

42. König IR, Fuchs O, Hansen G, von Mutius E, Kopp MV. What is Precision Medicine? Eur Respir J (2017) 50(4):1700391. doi: 10.1183/13993003.003912017

43. Hansen MR, Okuda DT. Precision Medicine for Multiple Sclerosis Promotes Preventative Medicine. Ann New Y Acad Sci (2018) 1420(1):62-71. doi: $10.1111 /$ nyas. 13846

44. Fagherazzi G. Deep Digital Phenotyping and Digital Twins for Precision Health: Time to Dig Deeper. J Med Internet Res (2020) 22(3):e16770. doi: $10.2196 / 16770$

45. Corral-Acero J, Margara F, Marciniak M, Rodero C, Loncaric F, Feng Y, et al. The 'Digital Twin' to Enable the Vision of Precision Cardiology. Eur Heart J (2020) 41:4556-64. doi: 10.1093/eurheartj/ehaa159

46. Bruynseels K, Santoni de Sio F, van den Hoven J. Digital Twins in Health Care: Ethical Implications of an Emerging Engineering Paradigm. Front Genet (2018) 9:31-. doi: 10.3389/fgene.2018.00031

47. Mahler M, Martinez-Prat L, Sparks JA, Deane KD. Precision Medicine in the Care of Rheumatoid Arthritis: Focus on Prediction and Prevention of Future Clinically-Apparent Disease. Autoimmun Rev (2020) 19(5):102506. doi: 10.1016/j.autrev.2020.102506

48. Björnsson B, Borrebaeck C, Elander N, Gasslander T, Gawel DR, Gustafsson M, et al. Digital Twins to Personalize Medicine. Genome Med (2019) 12 (1):4-. doi: 10.1186/s13073-019-0701-3

49. Ienca M, Ferretti A, Hurst S, Puhan M, Lovis C, Vayena E. Considerations for Ethics Review of Big Data Health Research: A Scoping Review. PloS One (2018) 13(10):e0204937. doi: 10.1371/journal.pone.0204937

50. Ziegelstein RC. Personomics: The Missing Link in the Evolution From Precision Medicine to Personalized Medicine. J Personalized Med (2017) 7 (4):11. doi: 10.3390/jpm7040011

51. Robinson PN. Deep Phenotyping for Precision Medicine. Hum Mutat (2012) 33(5):777-80. doi: 10.1002/humu.22080

52. Dorsey ER, Omberg L, Waddell E, Adams JL, Adams R, Ali MR, et al. Deep Phenotyping of Parkinson's Disease. J Parkinson's Dis (2020) 10(3):855-73. doi: 10.3233/jpd-202006

53. Delude CM. Deep Phenotyping: The Details of Disease. Nature (2015) 527 (7576):S14-5. doi: 10.1038/527S14a

54. Rieckmann P, Centonze D, Elovaara I, Giovannoni G, Havrdova E, Kesselring J, et al. Unmet Needs, Burden of Treatment, and Patient Engagement in Multiple Sclerosis: A Combined Perspective From the MS in the 21st Century Steering Group. Mult Scler Relat Disord (2018) 19:15360. doi: 10.1016/j.msard.2017.11.013 
55. Lin X, Yu M, Jelinek GA, Simpson-Yap S, Neate S, Nag N. Greater Engagement With Health Information Is Associated With Adoption and Maintenance of Healthy Lifestyle Behaviours in People With MS. Int J Environ Res Public Health (2020) 17(16):5935. doi: 10.3390/ijerph17165935

56. Gourraud PA, Henry RG, Cree BA, Crane JC, Lizee A, Olson MP, et al. Precision Medicine in Chronic Disease Management: The Multiple Sclerosis Bioscreen. Ann Neurol (2014) 76(5):633-42. doi: 10.1002/ana.24282

57. Brück W, Gold R, Lund BT, Oreja-Guevara C, Prat A, Spencer CM, et al. Therapeutic Decisions in Multiple Sclerosis: Moving Beyond Efficacy. JAMA Neurol (2013) 70(10):1315-24. doi: 10.1001/jamaneurol.2013.3510

58. Gafson A, Craner MJ, Matthews PM. Personalised Medicine for Multiple Sclerosis Care. Mult Scler (2017) 23(3):362-9. doi: 10.1177/ 1352458516672017

59. Chitnis T, Prat A. A Roadmap to Precision Medicine for Multiple Sclerosis. Multiple Sclerosis J (2020) 26(5):522-32. doi: 10.1177/1352458519881558

60. Bose G, Freedman MS. Precision Medicine in the Multiple Sclerosis Clinic: Selecting the Right Patient for the Right Treatment. Mult Scler (2020) 26 (5):540-7. doi: 10.1177/1352458519887324

61. Comabella M, Sastre-Garriga J, Montalban X. Precision Medicine in Multiple Sclerosis: Biomarkers for Diagnosis, Prognosis, and Treatment Response. Curr Opin Neurol (2016) 29(3):254-62. doi: 10.1097/ WCO.0000000000000336

62. Golan D, Staun-Ram E, Miller A. Shifting Paradigms in Multiple Sclerosis: From Disease-Specific, Through Population-Specific Toward PatientSpecific. Curr Opin Neurol (2016) 29(3):354-61. doi: 10.1097/ WCO.0000000000000324

63. Pulido-Valdeolivas I, Zubizarreta I, Martinez-Lapiscina EH, Villoslada P. Precision Medicine for Multiple Sclerosis: An Update of the Available Biomarkers and Their Use in Therapeutic Decision Making. Expert Rev Precis Med Drug Dev (2017) 2(6):345-61. doi: 10.1080/23808993.2017.1393315

64. Tao F, Zhang M, Nee AYC. Chapter 1 - Background and Concept of Digital Twin. In: F Tao, M Zhang and AYC Nee, editors. Digital Twin Driven Smart Manufacturing. London: Academic Press (2019). p. 3-28.

65. Grieves M. (2018). Available at: http://www.apriso.com/library/ Whitepaper_Dr_Grieves_DigitalTwin_ManufacturingExcellence. phphttp://www.apriso.com/library/Whitepaper_Dr_Grieves_DigitalTwin_ ManufacturingExcellence.php.

66. Rasheed A, San O, Kvamsdal T. Digital Twin: Values, Challenges and Enablers From a Modeling Perspective. IEEE Access (2020) 8:21980-2012. doi: 10.1109/ACCESS.2020.2970143

67. Tao F, Qi QL. Make More Digital Twins. Nature (2019) 573(7775):490-1. doi: 10.1038/d41586-019-02849-1

68. Chen Y. Integrated and Intelligent Manufacturing: Perspectives and Enablers. Engineering (2017) 3(5):588-95. doi: 10.1016/J.ENG.2017.04.009

69. Liu Z, Meyendorf N, Mrad N. The Role of Data Fusion in Predictive Maintenance Using Digital Twin. AIP Conf Proc (2018) 1949(1):020023. doi: $10.1063 / 1.5031520$

70. Zheng Y, Yang S, Cheng H. An Application Framework of Digital Twin and its Case Study. J Ambient Intell Humanized Computing (2019) 10(3):114153. doi: 10.1007/s12652-018-0911-3

71. Vrabič R, Erkoyuncu JA, Butala P, Roy R. Digital Twins: Understanding the Added Value of Integrated Models for Through-Life Engineering Services. Proc Manufacturing (2018) 16:139-46. doi: 10.1016/j.promfg.2018.10.167

72. Madni AM, Madni CC, Lucero SD. Leveraging Digital Twin Technology in Model-Based Systems Engineering. Systems (2019) 7(1):7. doi: 10.3390/ systems 7010007

73. Siemens. (2020). Available at: https://new.siemens.com/global/en/company/ stories/industry/the-digital-twin.htmlhttps://new.siemens.com/global/en/ company/stories/industry/the-digital-twin.html.

74. Cimino C, Negri E, Fumagalli L. Review of Digital Twin Applications in Manufacturing. Comput Industry (2019) 113:103130. doi: 10.1016/ j.compind.2019.103130

75. Kritzinger W, Karner M, Traar G, Henjes J, Sihn W. Digital Twin in Manufacturing: A Categorical Literature Review and Classification. IFACPapersOnLine (2018) 51(11):1016-22. doi: 10.1016/j.ifacol.2018.08.474

76. Digital Twin Driven Smart Manufacturing. (2019).

77. Boschert S, Rosen R. Digital Twin-The Simulation Aspect. In: P Hehenberger and D Bradley, editors. Mechatronic Futures: Challenges and
Solutions for Mechatronic Systems and Their Designers. Cham: Springer International Publishing (2016). p. 59-74.

78. Tao F, Cheng J, Qi Q, Zhang M, Zhang H, Sui F. Digital Twin-Driven Product Design, Manufacturing and Service With Big Data. Int J Advanced Manufacturing Technol (2018) 94(9):3563-76. doi: 10.1007/s00170-0170233-1

79. Urbina Coronado PD, Lynn R, Louhichi W, Parto M, Wescoat E, Kurfess T. Part Data Integration in the Shop Floor Digital Twin: Mobile and Cloud Technologies to Enable a Manufacturing Execution System. J Manufacturing Syst (2018) 48:25-33. doi: 10.1016/j.jmsy.2018.02.002

80. Schluse M, Priggemeyer M, Atorf L, Roßmann J. Experimentable Digital Twin" Streamlining Simulation-Based Systems Engineering for Industry 4.0. IEEE Trans Ind Inf (2018) 14:1722-31. doi: 10.1109/TII.2018.2804917

81. Laaki H, Miché Y, Tammi K. Prototyping a Digital Twin for Real Time Remote Control Over Mobile Networks: Application of Remote Surgery. IEEE Access (2019) 7:20325-36. doi: 10.1109/ACCESS.2019.2897018

82. Jimenez JI, Jahankhani H, Kendzierskyj S. Health Care in the Cyberspace: Medical Cyber-Physical System and Digital Twin Challenges. In: M Farsi, A Daneshkhah, A Hosseinian-Far and H Jahankhani, editors. Digital Twin Technologies and Smart Cities. Cham: Springer International Publishing (2020). p. 79-92.

83. Tao F, Zhang H, Liu A, Nee A. Digital Twin in Industry: State-of-the-Art. IEEE Trans Ind Inf (2019) 15:2405-15. doi: 10.1109/TII.2018.2873186

84. Kannadasan K, Edla DR, Kuppili V. Type 2 Diabetes Data Classification Using Stacked Autoencoders in Deep Neural Networks. Clin Epidemiol Global Health (2019) 7(4):530-5. doi: 10.1016/j.cegh.2018.12.004

85. Schroeder GN, Steinmetz C, Pereira CE, Espindola DB. Digital Twin Data Modeling With AutomationML and a Communication Methodology for Data Exchange. IFAC-PapersOnLine (2016) 49(30):12-7. doi: 10.1016/ j.ifacol.2016.11.115

86. Haag S, Anderl R. Digital Twin - Proof of Concept. Manufacturing Lett (2018) 15:64-6. doi: 10.1016/j.mfglet.2018.02.006

87. Uhlemann THJ, Schock C, Lehmann C, Freiberger S, Steinhilper R. The Digital Twin: Demonstrating the Potential of Real Time Data Acquisition in Production Systems. Proc Manufacturing (2017) 9:113-20. doi: 10.1016/ j.promfg.2017.04.043

88. Tao F, Zhang M, Liu Y, Nee AYC. Digital Twin Driven Prognostics and Health Management for Complex Equipment. Cirp Ann-Manuf Techn (2018) 67:169-72. doi: 10.1016/j.cirp.2018.04.055

89. Hirsch MC. Warum Intelligente Decision-Support-Systeme Das Betriebssystem Eines Smart Hospitals Sein Und Medizin Menschlicher Machen Werden. In: JA Werner, M Forsting, T Kaazte and A SchmidtRumposch, editors. Smart Hospital - Digitale Und Empathische Zukunftsmedizin. Berlin: MMV Medizinisch Wissenschaftliche Verlagsgesellschaft (2020). p. 93-102.

90. Shameer K, Johnson KW, Glicksberg BS, Dudley JT, Sengupta PP. Machine Learning in Cardiovascular Medicine: Are We There Yet? Heart (2018) 104 (14):1156-64. doi: 10.1136/heartjnl-2017-311198

91. Rajula HSR, Verlato G, Manchia M, Antonucci N, Fanos V. Comparison of Conventional Statistical Methods With Machine Learning in Medicine: Diagnosis, Drug Development, and Treatment. Med (Kaunas Lithuania) (2020) 56(9):455. doi: 10.3390/medicina56090455

92. Winter NR, Hahn T. Big Data, AI and Machine Learning for Precision Psychiatry: How are They Changing the Clinical Practice? Fortschr Der Neurol-Psychiatr (2020) 88(12):786-93. doi: 10.1055/a-1234-6247

93. Alber M, Buganza Tepole A, Cannon WR, De S, Dura-Bernal S, Garikipati K, et al. Integrating Machine Learning and Multiscale Modeling-Perspectives, Challenges, and Opportunities in the Biological, Biomedical, and Behavioral Sciences. NPJ Digit Med (2019) 2(1):115. doi: 10.1038/s41746-019-0193-y

94. Rao D, Mane S. Digital Twin Approach to Clinical DSS With Explainable Ai. (2019) Available at: https://arxiv.org/abs/1910.13520v1.

95. Digital Twins in Healthcare. (2020). Available at: https://www.persistent. com/whitepaper-digital-twins-in-healthcare/.

96. Digitwins. (2018). Available at: https://www.digitwins.orghttps://www. digitwins.org.

97. Filippo MD, Damiani C, Vanoni M, Maspero D, Mauri G, Alberghina L, et al. Single-Cell Digital Twins for Cancer Preclinical Investigation. Methods Mol Biol (Clifton NJ) (2020) 2088:331-43. doi: 10.1007/978-1-0716-0159-4_15 
98. Ardila D, Kiraly AP, Bharadwaj S, Choi B, Reicher JJ, Peng L, et al. End-toEnd Lung Cancer Screening With Three-Dimensional Deep Learning on Low-Dose Chest Computed Tomography. Nat Med (2019) 25(6):954-61. doi: 10.1038/s41591-019-0447-x

99. Wilhelm D, Berlet M, Feussner H, Ostler D. Digitalisierung in Der Onkologischen Chirurgie. Forum (2021) 36:22-8. doi: 10.1007/s12312020-00879-9

100. Zhang J, Qian H, Zhou H. Application and Research of Digital Twin Technology in Safety and Health Monitoring of the Elderly in Community. Zhongguo Yi Liao Qi Xie Za Zhi Chin J Med Instrumentation (2019) 43(6):410-3. doi: 10.3969/j.issn.1671-7104.2019.06.005

101. Calderita LV, Vega A, Barroso-Ramírez S, Bustos P, Núñez P. Designing a Cyber-Physical System for Ambient Assisted Living: A Use-Case Analysis for Social Robot Navigation in Caregiving Centers. Sensors (Basel Switzerland) (2020) 20(14):4005. doi: 10.3390/s20144005

102. Hirschvogel M, Jagschies L, Maier A, Wildhirt SM, Gee MW. An in Silico Twin for Epicardial Augmentation of the Failing Heart. Int J Numerical Methods Biomed Eng (2019) 35(10):e3233. doi: 10.1002/cnm.3233

103. Hose DR, Lawford PV, Huberts W, Hellevik LR, Omholt SW, van de Vosse FN. Cardiovascular Models for Personalised Medicine: Where Now and Where Next? Med Eng Phys (2019) 72:38-48. doi: 10.1016/ j.medengphy.2019.08.007

104. Mazumder O, Roy D, Bhattacharya S, Sinha A, Pal A eds. In: 201941 st Annual International Conference of the IEEE Engineering in Medicine and Biology Society (EMBC). (2019) 5024-29. doi: 10.1016/j.medengphy.2019.08.00710.1109/ EMBC.2019.8856691

105. Niederer SA, Aboelkassem Y, Cantwell CD, Corrado C, Coveney S, Cherry EM, et al. Creation and Application of Virtual Patient Cohorts of Heart Models. Philos Trans A Math Phys Eng Sci (2020) 378:20190558. doi: 10.1098/rsta.2019.0558

106. Sharma P, Suehling M, Flohr T, Comaniciu D. Artificial Intelligence in Diagnostic Imaging: Status Quo, Challenges, and Future Opportunities. $J$ Thoracic Imaging (2020) 35 Suppl 1:S11-s6. doi: 10.1097/ RTI.0000000000000499

107. Ivanov D. Predicting the Impacts of Epidemic Outbreaks on Global Supply Chains: A Simulation-Based Analysis on the Coronavirus Outbreak (COVID-19/SARS-CoV-2) Case. Transportation Res Part E Logistics Transport Rev (2020) 136:101922. doi: 10.1016/j.tre.2020.101922

108. Tellechea-Luzardo J, Winterhalter C, Widera P, Kozyra J, de Lorenzo V, Krasnogor N. Linking Engineered Cells to Their Digital Twins: A Version Control System for Strain Engineering. ACS Synthetic Biol (2020) 9(3):53645. doi: 10.1021/acssynbio.9b00400

109. Lauzeral N, Borzacchiello D, Kugler M, George D, Rémond Y, Hostettler A, et al. A Model Order Reduction Approach to Create Patient-Specific Mechanical Models of Human Liver in Computational Medicine Applications. Comput Methods Programs Biomed (2019) 170:95-106. doi: 10.1016/j.cmpb.2019.01.003

110. Tomašev N, Glorot X, Rae JW, Zielinski M, Askham H, Saraiva A, et al. A Clinically Applicable Approach to Continuous Prediction of Future Acute Kidney Injury. Nature (2019) 572(7767):116-9. doi: 10.1038/s41586-019$1390-1$

111. Pizzolato C, Saxby DJ, Palipana D, Diamond LE, Barrett RS, Teng YD, et al. Neuromusculoskeletal Modeling-Based Prostheses for Recovery After Spinal Cord Injury. Front Neurorobot (2019) 13:97-. doi: 10.3389/fnbot.2019.00097

112. Chakshu NK, Carson J, Sazonov I, Nithiarasu P. A Semi-Active Human Digital Twin Model for Detecting Severity of Carotid Stenoses From Head Vibration-a Coupled Computational Mechanics and Computer Vision Method. Int J Numerical Methods Biomed Eng (2019) 35(5):e3180-e. doi: $10.1002 / \mathrm{cnm} .3180$

113. Lareyre F, Adam C, Carrier M, Raffort J. Using Digital Twins for Precision Medicine in Vascular Surgery. Ann Vasc Surg (2020) 5096(20):30379-4. doi: 10.1016/j.avsg.2020.04.042

114. Topol EJ. High-Performance Medicine: The Convergence of Human and Artificial Intelligence. Nat Med (2019) 25(1):44-56. doi: 10.1038/s41591018-0300-7

115. The living heart project. (2020). Available at: https://www.3ds.com/productsservices/simulia/solutions/life-sciences/the-living-heart-project/https:// www.3ds.com/products-services/simulia/solutions/life-sciences/the-livingheart-project/.
116. Grätzel von Grätz P. (2019). Available at: https://www.siemens-healthineers. $\mathrm{com} / \mathrm{dk} /$ news/mso-solutions-for-individual-patients.htmlhttps://www. siemens-healthineers.com/dk/news/mso-solutions-for-individual-patients. html.

117. Barricelli BR, Casiraghi E, Fogli D. A Survey on Digital Twin: Definitions, Characteristics, Applications, and Design Implications. IEEE Access (2019) 7:167653-71. doi: 10.1109/ACCESS.2019.2953499

118. Walsh JR, Smith AM, Pouliot Y, Li-Bland D, Loukianov A, Fisher CK. Generating Digital Twins With Multiple Sclerosis Using Probabilistic Neural Networks. (2020). doi: 10.1101/2020.02.04.934679

119. D Petrova-Antonova, I Spasov, I Krasteva, I Manova and S Ilieva eds. A Digital Twin Platform for Diagnostics and Rehabilitation of Multiple Sclerosis. Cham: Springer International Publishing (2020).

120. Fuller A, Fan Z, Day C. Digital Twin: Enabling Technologies, Challenges and Open Research. IEEE Access (2020) 8:108952-71. doi: 10.1109/ ACCESS.2020.2998358

121. Ziemssen T, Hillert J, Butzkueven H. The Importance of Collecting Structured Clinical Information on Multiple Sclerosis. BMC Med (2016) 14:81. doi: 10.1186/s12916-016-0627-1

122. Magyari M, Sorensen PS. Comorbidity in Multiple Sclerosis. Front Neurol (2020) 11(851). doi: 10.3389/fneur.2020.00851

123. Toscano S, Patti F. CSF Biomarkers in Multiple Sclerosis: Beyond Neuroinflammation. Neuroimmunol Neuroinflamm (2020) 7:14-41. doi: 10.20517/2347-8659.2020.12

124. Ziemssen T, Akgün K, Brück W. Molecular Biomarkers in Multiple Sclerosis. J Neuroinflamm (2019) 16(1):272. doi: 10.1186/s12974-019-1674-2

125. Thebault S, Booth RA, Freedman MS. Blood Neurofilament Light Chain: The Neurologist's Troponin? Biomedicines (2020) 8(11):523. doi: 10.3390/ biomedicines 8110523

126. Ziemssen T, Piani-Meier D, Bennett B, Johnson C, Tinsley K, Trigg A, et al. A Physician-Completed Digital Tool for Evaluating Disease Progression (Multiple Sclerosis Progression Discussion Tool): Validation Study. J Med Internet Res (2020) 22(2):e16932. doi: 10.2196/16932

127. D'Souza M, Yaldizli Ö, John R, Vogt DR, Papadopoulou A, Lucassen E, et al. Neurostatus e-Scoring Improves Consistency of Expanded Disability Status Scale Assessments: A Proof of Concept Study. Mult Scler (2017) 23(4):597603. doi: $10.1177 / 1352458516657439$

128. Kosa P, Barbour C, Wichman A, Sandford M, Greenwood M, Bielekova B. NeurEx: Digitalized Neurological Examination Offers a Novel HighResolution Disability Scale. Ann Clin Trans Neurol (2018) 5(10):1241-9. doi: $10.1002 / \mathrm{acn} 3.640$

129. Kurtzke JFM. Rating Neurologic Impairment in Multiple Sclerosis: An Expanded Disability Status Scale (EDSS). Neurology (1983) 33(11):144452. doi: 10.1212/WNL.33.11.1444

130. Beste C, Mückschel M, Paucke M, Ziemssen T. Dual-Tasking in Multiple Sclerosis - Implications for a Cognitive Screening Instrument. Front Hum Neurosci (2018) 12:24. doi: 10.3389/fnhum.2018.00024

131. Trentzsch K, Weidemann ML, Torp C, Inojosa H, Scholz M, Haase R, et al. The Dresden Protocol for Multidimensional Walking Assessment (DMWA) in Clinical Practice. Front Neurosci (2020) 14:582046. doi: 10.3389/ fnins.2020.582046

132. Lublin FD, Reingold SC, Cohen JA, Cutter GR, Sørensen PS, Thompson AJ, et al. Defining the Clinical Course of Multiple Sclerosis. 2013 Revisions (2014) 83(3):278-86. doi: 10.1212/wnl.46.4.907

133. De Meo E, Portaccio E, Giorgio A, Ruano L, Goretti B, Niccolai C, et al. Identifying the Distinct Cognitive Phenotypes in Multiple Sclerosis. JAMA Neurol (2021)78(4):414-425. doi: 10.1001/jamaneurol. 2020.4920

134. Inojosa H, Schriefer D, Ziemssen T. Clinical Outcome Measures in Multiple Sclerosis: A Review. Autoimmun Rev (2020) 19(5):102512. doi: 10.1016/ j.autrev.2020.102512

135. D’Souza M, Papadopoulou A, Girardey C, Kappos L. Standardization and Digitization of Clinical Data in Multiple Sclerosis. Nat Rev Neurol (2021):119-125. doi: 10.1038/s41582-020-00448-7

136. Meyer zu Hörste G, Gross CC, Klotz L, Schwab N, Wiendl H. NextGeneration Neuroimmunology: New Technologies to Understand Central Nervous System Autoimmunity. Trends Immunol (2020)41(4):341-354. doi: 10.1016/j.it.2020.02.005 
137. Leocani L, Rocca MA, Comi G. MRI and Neurophysiological Measures to Predict Course, Disability and Treatment Response in Multiple Sclerosis. Curr Opin Neurol (2016) 29(3):243-53. doi: 10.1097/WCO.0000000000000333

138. Marciniewicz E, Podgórski P, Sąsiadek M, Bladowska J. The Role of MR Volumetry in Brain Atrophy Assessment in Multiple Sclerosis: A Review of the Literature. Adv Clin Exp Med Off Organ Wroclaw Med Univ (2019) 28 (7):989-99. doi: 10.17219/acem/94137

139. Louapre C. Conventional and Advanced MRI in Multiple Sclerosis. Rev Neurol (2018) 174(6):391-7. doi: 10.1016/j.neurol.2018.03.009

140. Kaufmann M, Haase R, Proschmann U, Ziemssen T, Akgün K. Real-World Lab Data in Natalizumab Treated Multiple Sclerosis Patients Up to 6 Years LongTerm Follow Up. Front Neurol (2018) 9:1071. doi: 10.3389/fneur.2018.01071

141. Kaufmann M, Haase R, Proschmann U, Ziemssen T, Akgün K. Real World Lab Data: Patterns of Lymphocyte Counts in Fingolimod Treated Patients. Front Immunol (2018) 9:2669. doi: 10.3389/fimmu.2018.02669

142. Barro C, Chitnis T, Weiner HL. Blood Neurofilament Light: A Critical Review of its Application to Neurologic Disease. Ann Clin Trans Neurol (2020) 7(12):2508-23. doi: 10.1002/acn3.51234

143. Akgün K, Kretschmann N, Haase R, Proschmann U, Kitzler HH, Reichmann $\mathrm{H}$, et al. Profiling Individual Clinical Responses by High-Frequency Serum Neurofilament Assessment in MS. Neurol(R) Neuroimmunol Neuroinflamm (2019) 6(3):e555. doi: 10.1212/NXI.0000000000000555

144. Cortese R, Collorone S, Ciccarelli O, Toosy AT. Advances in Brain Imaging in Multiple Sclerosis. Ther Adv Neurol Disord (2019) 121-15. doi: 10.1177/ 1756286419859722

145. Oreja-Guevara C. Overview of Magnetic Resonance Imaging for Management of Relapsing-Remitting Multiple Sclerosis in Everyday Practice. Eur J Neurol (2015) 22 Suppl 2:22-7. doi: 10.1111/ene.12800

146. Tomassini V, Sinclair A, Sawlani V, Overell J, Pearson OR, Hall J, et al. Diagnosis and Management of Multiple Sclerosis: MRI in Clinical Practice. J Neurol (2020) 267(10):2917-25. doi: 10.1007/s00415-020-09930-0

147. Oh J, Sicotte NL. New Imaging Approaches for Precision Diagnosis and Disease Staging of MS? Mult Scler (2020) 26(5):568-75. doi: 10.1177/1352458519871817

148. Arevalo O, Riascos R, Rabiei P, Kamali A, Nelson F. Standardizing Magnetic Resonance Imaging Protocols, Requisitions, and Reports in Multiple Sclerosis: An Update for Radiologist Based on 2017 Magnetic Resonance Imaging in Multiple Sclerosis and 2018 Consortium of Multiple Sclerosis Centers Consensus Guidelines. J Comput Assisted Tomography (2019) 43 (1):1-12. doi: 10.1097/rct.0000000000000767

149. Saslow L, Li DKB, Halper J, Banwell B, Barkhof F, Barlow L, et al. An International Standardized Magnetic Resonance Imaging Protocol for Diagnosis and Follow-up of Patients With Multiple Sclerosis: Advocacy, Dissemination, and Implementation Strategies. Int J MS Care (2020) 22 (5):226-32. doi: 10.7224/1537-2073.2020-094

150. Pessini RA, ACd S, Salmon CEG. Quantitative MRI Data in Multiple Sclerosis Patients: A Pattern Recognition Study. Res Biomed Eng (2018) 34:138-46. doi: 10.1590/2446-4740.07117

151. Afzal HMR, Luo S, Ramadan S, Lechner-Scott J. The Emerging Role of Artificial Intelligence in Multiple Sclerosis Imaging. Mult Scler (2020) 1-10. doi: $10.1177 / 1352458520966298$

152. Tauhid S, Neema M, Healy BC, Weiner HL, Bakshi R. MRI Phenotypes Based on Cerebral Lesions and Atrophy in Patients With Multiple Sclerosis. J Neurol Sci (2014) 346(1-2):250-4. doi: 10.1016/j.jns.2014.08.047

153. Hanson JVM, Wicki CA, Manogaran P, Petzold A, Schippling S. OCT and Imaging in Central Nervous System Diseases: The Eye as a Window to the Brain. In: OCT and Multiple Sclerosis, 2nd ed. (2020). p. 195-233. doi: 10.1007/978-3-030-26269-3_11

154. Bauckneht M, Capitanio S, Raffa S, Roccatagliata L, Pardini M, Lapucci C, et al. Molecular Imaging of Multiple Sclerosis: From the Clinical Demand to Novel Radiotracers. EJNMMI Radiopharmacy Chem (2019) 4(1):6. doi: 10.1186/s41181-019-0058-3

155. Dorsey ER, Papapetropoulos S, Xiong M, Kieburtz K. The First Frontier: Digital Biomarkers for Neurodegenerative Disorders. Digital Biomarkers (2017) 1(1):6-13. doi: 10.1159/000477383

156. Perry B, Herrington W, Goldsack JC, Grandinetti CA, Vasisht KP, Landray MJ, et al. Use of Mobile Devices to Measure Outcomes in Clinical Research, 2010-2016: A Systematic Literature Review. Digital Biomarkers (2018) 2 (1):11-30. doi: 10.1159/000486347
157. Kang C, Janes H, Tajik P, Groen H, Mol B, Koopmans C, et al. Evaluation of Biomarkers for Treatment Selection Using Individual Participant Data From Multiple Clinical Trials. Stat Med (2018) 37(9):1439-53. doi: 10.1002/ sim.7608

158. Dagum P. Digital Biomarkers of Cognitive Function. NPJ Digit Med (2018) 1 (1):10. doi: 10.1038/s41746-018-0018-4

159. Barrios L, Oldrati P, Santini S, Lutterotti A. (2018). Recognizing Digital Biomarkers for Fatigue Assessment in Patients with Multiple Sclerosis.

160. Rudick RA, Miller D, Bethoux F, Rao SM, Lee JC, Stough D, et al. The Multiple Sclerosis Performance Test (MSPT): An iPad-based Disability Assessment Tool. J Vis Exp (2014) 88):e51318. doi: 10.3791/51318

161. Rao SM, Galioto R, Sokolowski M, McGinley M, Freiburger J, Weber M, et al. Multiple Sclerosis Performance Test: Validation of Self-Administered Neuroperformance Modules. Eur J Neurol (2020) 27(5):878-86. doi: 10.1111/ene.14162

162. Rao SM, Losinski G, Mourany L, Schindler D, Mamone B, Reece C, et al. Processing Speed Test: Validation of a Self-Administered, iPad $\left({ }^{\circledR}\right)$-based Tool for Screening Cognitive Dysfunction in a Clinic Setting. Mult Scler (2017) 23(14):1929-37. doi: 10.1177/1352458516688955

163. Gijbels D, Eijnde BO, Feys P. Comparison of the 2- and 6-Minute Walk Test in Multiple Sclerosis. Mult Scler (2011) 17(10):1269-72. doi: 10.1177/ 1352458511408475

164. Rossier P, Wade DT. Validity and Reliability Comparison of 4 Mobility Measures in Patients Presenting With Neurologic Impairment. Arch Phys Med Rehabil (2001) 82(1):9-13. doi: 10.1053/apmr.2001.9396

165. Marziniak M, Brichetto G, Feys P, Meyding-Lamade U, Vernon K, Meuth SG. The Use of Digital and Remote Communication Technologies as a Tool for Multiple Sclerosis Management: Narrative Review. JMIR Rehabil Assist Technol (2018) 5(1):e5. doi: 10.2196/rehab.7805

166. Scholz M, Haase R, Schriefer D, Voigt I, Ziemssen T. Electronic Health Interventions in the Case of Multiple Sclerosis: From Theory to Practice. Brain Sci (2021) 11(2):180. doi: 10.3390/brainsci1 1020180

167. Babre D. Clinical Data Interchange Standards Consortium: A Bridge to Overcome Data Standardisation. Perspect Clin Res (2013) 4(2):115-6. doi: 10.4103/2229-3485.111779

168. Peeters LM, Parciak T, Kalra D, Moreau Y, Kasilingam E, van Galen P, et al. Multiple Sclerosis Data Alliance - A Global Multi-Stakeholder Collaboration to Scale-Up Real World Data Research. Multiple Sclerosis Related Disord (2021) 47:102634. doi: 10.1016/j.msard.2020.102634

169. Tilocca B, Pieroni L, Soggiu A, Britti D, Bonizzi L, Roncada P, et al. GutBrain Axis and Neurodegeneration: State-of-the-Art of Meta-Omics Sciences for Microbiota Characterization. Int J Mol Sci (2020) 21(11):4045. doi: $10.3390 /$ ijms 21114045

170. Martin NA, Nawrocki A, Molnar V, Elkjaer ML, Thygesen EK, Palkovits M, et al. Orthologous Proteins of Experimental De- and Remyelination are Differentially Regulated in the CSF Proteome of Multiple Sclerosis Subtypes. PloS One (2018) 13(8):e0202530. doi: 10.1371/journal.pone.0202530

171. Malekzadeh A, Teunissen C. Recent Progress in Omics-Driven Analysis of MS to Unravel Pathological Mechanisms. Expert Rev Neurother (2013) 13 (9):1001-16. doi: 10.1586/14737175.2013.835602

172. Sun YV, Hu Y-J. Integrative Analysis of Multi-omics Data for Discovery and Functional Studies of Complex Human Diseases. Adv Genet (2016) 93:14790. doi: 10.1016/bs.adgen.2015.11.004

173. Chase Huizar C, Raphael I, Forsthuber TG. Genomic, Proteomic, and Systems Biology Approaches in Biomarker Discovery for Multiple Sclerosis. Cell Immunol (2020) 358:104219. doi: 10.1016/j.cellimm.2020.104219

174. Subramanian I, Verma S, Kumar S, Jere A, Anamika K. Multi-Omics Data Integration, Interpretation, and Its Application. Bioinf Biol Insights (2020) 14:1177932219899051. doi: 10.1177/1177932219899051

175. Klose K, Kreimeier S, Tangermann U, Aumann I, Damm Kon behalf of the RHOG. Patient- and Person-Reports on Healthcare: Preferences, Outcomes, Experiences, and Satisfaction - an Essay. Health Econom Rev (2016) 6(1):18. doi: 10.1186/s13561-016-0094-6

176. D’Amico E, Haase R, Ziemssen T. Review: Patient-reported Outcomes in Multiple Sclerosis Care. Mult Scler Relat Disord (2019) 33:61-6. doi: 10.1016/ j.msard.2019.05.019

177. Medina LD, Torres S, Alvarez E, Valdez B, Nair KV. Patient-Reported Outcomes in Multiple Sclerosis: Validation of the Quality of Life in 
Neurological Disorders (Neuro-QoL ${ }^{\mathrm{TM}}$ ) Short Forms. Mult Scler J Exp Transl Clin (2019) 5(4):1-11. doi: 10.1177/2055217319885986

178. Cella D, Lai JS, Nowinski CJ, Victorson D, Peterman A, Miller D, et al. Neuro-QOL: Brief Measures of Health-Related Quality of Life for Clinical Research in Neurology. Neurology (2012) 78(23):1860-7. doi: 10.1212/ WNL.0b013e318258f744

179. Hobart JC, Riazi A, Lamping DL, Fitzpatrick R, Thompson AJ. Measuring the Impact of MS on Walking Ability: The 12-Item MS Walking Scale (Msws-12). Neurology (2003) 60(1):31-6. doi: 10.1212/wnl.60.1.31

180. Ziemssen T, Phillips G, Shah R, Mathias A, Foley C, Coon C, et al. Development of the Multiple Sclerosis (MS) Early Mobility Impairment Questionnaire (EMIQ). J Neurol (2016) 263(10):1969-83. doi: 10.1007/ s00415-016-8210-4

181. Hodson M, Andrew S, Michael Roberts C. Towards an Understanding of PREMS and PROMS in COPD. Breathe (2013) 9(5):358-64. doi: 10.1183/ 20734735.006813

182. Male L, Noble A, Atkinson J, Marson T. Measuring Patient Experience: A Systematic Review to Evaluate Psychometric Properties of Patient Reported Experience Measures (Prems) for Emergency Care Service Provision. Int $J$ Qual Health Care (2017) 29(3):314-26. doi: 10.1093/intqhc/mzx027

183. The Lancet N. Patient-Reported Outcomes in the Spotlight. Lancet Neurol (2019) 18(11):981. doi: 10.1016/S1474-4422(19)30357-6

184. Wiendl H, Korsukewitz C, Kieseier BC. Klinik, Diagnostik Und Therapie. Klinische Neurologie. In: Multiple Sklerose. Stuttgart: Kohlhammer (2021).

185. Giovannoni G, Bermel R, Phillips T, Rudick R. A Brief History of NEDA. Mult Scler Relat Disord (2018) 20:228-30. doi: 10.1016/j.msard.2017.07.011

186. Giovannoni G, Butzkueven HD-JS, Hobart J, Kobelt G, Pepper G, Sormani MP, et al. (2018). Brain Health: Keine Zeit verlieren bei Multipler Sklerose.

187. Linker R, Kallmann BA, Kleinschnitz C, Rieckmann P, Maurer M, Schwab S. "Time is Brain" in Relapsing Remitting Multiple Sclerosis. Curr Treat Concepts Immunother Nervenarzt (2015) 86(12):1528-37. doi: 10.1007/s00115-015-4439-X

188. Soelberg Sorensen P, Giovannoni G, Montalban X, Thalheim C, Zaratin P, Comi G. The Multiple Sclerosis Care Unit. Mult Scler (2018) 418:627-36. doi: $10.1177 / 1352458518807082$

189. Stühler E, Braune S, Lionetto F, Heer Y, Jules E, Westermann C, et al. Framework for Personalized Prediction of Treatment Response in Relapsing Remitting Multiple Sclerosis. BMC Med Res Methodol (2020) 20(1):24. doi: 10.1186/s12874-020-0906-6

190. Kalincik T, Manouchehrinia A, Sobisek L, Jokubaitis V, Spelman T, Horakova D, et al. Towards Personalized Therapy for Multiple Sclerosis: Prediction of Individual Treatment Response. Brain (2017) 140(9):2426-43. doi: 10.1093/brain/awx185

191. Kalincik T. Reply: Towards Personalized Therapy for Multiple Sclerosis: Limitations of Observational Data. Brain (2018)141(5):e39. doi: 10.1093/ brain/awy056

192. Pinto MF, Oliveira H, Batista S, Cruz L, Pinto M, Correia I, et al. Prediction of Disease Progression and Outcomes in Multiple Sclerosis With Machine Learning. Sci Rep (2020) 10(1):21038. doi: 10.1038/s41598-020-78212-6

193. HL7 FHIR. (2017). Available at: http://hl7.org/fhir/http://hl7.org/fhir/.

194. XDS Value Sets für Deutschland. (2018). Available at: http://www.ihe-d.de/ projekte/xds-value-sets-fuer-deutschland/http://www.ihe-d.de/projekte/xdsvalue-sets-fuer-deutschland/.

195. Voigt I, Benedict M, Susky M, Scheplitz T, Frankowitz S, Kern R, et al. A Digital Patient Portal for Patients With Multiple Sclerosis. Front Neurol (2020) 11(400). doi: 10.3389/fneur.2020.00400

196. Benedict M, Schlieter H, Burwitz M, Scheplitz T, Susky M, Richter P, et al. Patientenintegration Durch Pfadsysteme. Wirtschaftsinformatik (2019). Siegen. Available at: https://www.researchgate.net/publication/ 330203014_Patientenintegration_durch_Pfadsysteme.

197. Benedict P, Schlieter H. Understanding Patient Pathways in the Context of Integrated Health Care Services - Implications From a Scoping Review. 14 Internationalen Tagung Wirtschaftsinformatik (2019). Siegen. Available at: https://www.semanticscholar.org/paper/Understanding-Patient-Pathways-inthe-Context-of-a-Richter-Schlieter/dca3766d0c79a152da2f62421cae f03ce455e96c.
198. Minkman M, Ahaus K, Fabbricotti I, Nabitz U, Huijsman R. A Quality Management Model for Integrated Care: Results of a Delphi and Concept Mapping Study. Int J Qual Health Care (2009) 21(1):66-75. doi: 10.1093/ intqhe/mzn048

199. Multiple sclerosis. (2016). Available at: https://www.nice.org.uk/guidance/ qs108https://www.nice.org.uk/guidance/qs108.

200. Patient experience in adult NHS services. (2012). Available at: https://www. nice.org.uk/guidance/qs15https://www.nice.org.uk/guidance/qs15.

201. Haase R, Wunderlich M, Dillenseger A, Kern R, Akgun K, Ziemssen T. Improving Multiple Sclerosis Management and Collecting Safety Information in the Real World: The MSDS3D Software Approach. Expert Opin Drug Saf (2018) 17(4):369-78. doi: 10.1080/14740338.2018.1437144

202. Ziemssen T, Kern R, Voigt I, Haase R. Data Collection in Multiple Sclerosis: The Msds Approach. Front Neurol (2020) 11(445). doi: 10.3389/fneur.2020.00445

203. Schleimer E, Pearce J, Barnecut A, Rowles W, Lizee A, Klein A, et al. A Precision Medicine Tool for Patients With Multiple Sclerosis (the Open Ms BioScreen): Human-Centered Design and Development. J Med Internet Res (2020) 22(7):e15605. doi: 10.2196/15605

204. Shortliffe EH, Sepúlveda MJ. Clinical Decision Support in the Era of Artificial Intelligence. Jama (2018) 320(21):2199-200. doi: 10.1001/jama.2018.17163

205. Sutton RT, Pincock D, Baumgart DC, Sadowski DC, Fedorak RN, Kroeker KI. An Overview of Clinical Decision Support Systems: Benefits, Risks, and Strategies for Success. NPJ Digit Med (2020) 3:17. doi: 10.1038/s41746-020-0221-y

206. Alshamrani R, Althbiti A, Alshamrani Y, Alkomah F, Ma X. Model-Driven Decision Making in Multiple Sclerosis Research: Existing Works and Latest Trends. Patterns (N YNY) (2020) 1(8):100121. doi: 10.1016/j.patter.2020.100121

207. Zhao Y, Healy BC, Rotstein D, Guttmann CR, Bakshi R, Weiner HL, et al. Exploration of Machine Learning Techniques in Predicting Multiple Sclerosis Disease Course. PloS One (2017) 12(4):e0174866. doi: 10.1371/ journal.pone.0174866

208. Zhao Y, Wang T, Bove R, Cree B, Henry R, Lokhande H, et al. Ensemble Learning Predicts Multiple Sclerosis Disease Course in the SUMMIT Study. NPJ Digit Med (2020) 3:135. doi: 10.1038/s41746-020-00361-9

209. Goyal M, Khanna D, Rana PS, Khaibullin T, Martynova E, Rizvanov AA, et al. Computational Intelligence Technique for Prediction of Multiple Sclerosis Based on Serum Cytokines. Front Neurol (2019) 10:781. doi: 10.3389/fneur.2019.00781

210. Deans KJ, Sabihi S, Forrest CB. Learning Health Systems. Semin Pediatr Surg (2018) 27(6):375-8. doi: 10.1053/j.sempedsurg.2018.10.005

211. Warraich HJ, Califf RM, Krumholz HM. The Digital Transformation of Medicine can Revitalize the Patient-Clinician Relationship. NPJ Digit Med (2018) 1:49. doi: 10.1038/s41746-018-0060-2

212. Verghese A, Shah NH, Harrington RA. What This Computer Needs is a Physician: Humanism and Artificial Intelligence. JAMA (2018) 319(1):1920. doi: 10.1001/jama.2017.19198

213. Nordling L. A Fairer Way Forward for AI in Health Care. Nature (2019) 573 (7775):S103-s5. doi: 10.1038/d41586-019-02872-2

214. Asan O, Bayrak AE, Choudhury A. Artificial Intelligence and Human Trust in Healthcare: Focus on Clinicians. J Med Internet Res (2020) 22(6):e15154. doi: $10.2196 / 15154$

215. Bhattad PB, Jain V. Artificial Intelligence in Modern Medicine - The Evolving Necessity of the Present and Role in Transforming the Future of Medical Care. Cureus (2020) 12(5):e8041. doi: 10.2196/preprints.18829

Conflict of Interest: The authors declare that the research was conducted in the absence of any commercial or financial relationships that could be construed as a potential conflict of interest.

Copyright $\odot 2021$ Voigt, Inojosa, Dillenseger, Haase, Akgün and Ziemssen. This is an open-access article distributed under the terms of the Creative Commons Attribution License (CC BY). The use, distribution or reproduction in other forums is permitted, provided the original author(s) and the copyright owner(s) are credited and that the original publication in this journal is cited, in accordance with accepted academic practice. No use, distribution or reproduction is permitted which does not comply with these terms. 